\title{
PHYSICAL AND MORPHOLOGICAL PROPERTIES OF [O II] EMITTING GALAXIES IN THE HETDEX PILOT SURVEY
}

\author{
Joanna S. Bridge ${ }^{1,5}$, Caryl Gronwall ${ }^{1,5}$, Robin Ciardullo $^{1,5}$, Alex Hagen $^{1,5}$, Greg ZeimanN $^{1,5}$, A. I. Malz $^{1,4,5}$, \\ Viviana Acquaviva ${ }^{2}$, Donald P. Schneider ${ }^{1,5}$, Niv Drory ${ }^{3}$, Karl GebhardT $^{3}$, and Shardha Jogee ${ }^{3}$ \\ ${ }^{1}$ Department of Astronomy \& Astrophysics, The Pennsylvania State University, University Park, PA 16802, USA; jsbridge@ psu.edu, \\ caryl@astro.psu.edu, rbc@astro.psu.edu, hagen@psu.edu, grzeimann@psu.edu, aimalz@psu.edu,dps@astro.psu.edu \\ ${ }^{2}$ Department of Physics, New York City College of Technology, City University of New York, Brooklyn, NY 11201, USA; vacquaviva@citytech.cuny.edu \\ ${ }^{3}$ Department of Astronomy, University of Texas at Austin, Austin, TX 78712, USA; drory@astro.as.utexas.edu, \\ gebhardt@astro.as.utexas.edu, sj@astro.as.utexas.edu \\ ${ }^{4}$ Center for Cosmology and Particle Physics, Department of Physics, New York University, New York, NY 10003 \\ Received 2013 December 18; accepted 2014 November 18; published 2015 January 30
}

\begin{abstract}
The Hobby-Eberly Dark Energy Experiment pilot survey identified 284 [O II] $\lambda 3727$ emitting galaxies in a $169 \operatorname{arcmin}^{2}$ field of sky in the redshift range $0<z<0.57$. This line flux limited sample provides a bridge between studies in the local universe and higher-redshift [O II] surveys. We present an analysis of the star formation rates (SFRs) of these galaxies as a function of stellar mass as determined via spectral energy distribution fitting. The [O II] emitters fall on the "main sequence" of star-forming galaxies with SFR decreasing at lower masses and redshifts. However, the slope of our relation is flatter than that found for most other samples, a result of the metallicity dependence of the [O II] star formation rate indicator. The mass-specific SFR is higher for lower mass objects, supporting the idea that massive galaxies formed more quickly and efficiently than their lower mass counterparts. This is confirmed by the fact that the equivalent widths of the [O II] emission lines trend smaller with larger stellar mass. Examination of the morphologies of the [O II] emitters reveals that their star formation is not a result of mergers, and the galaxies' half-light radii do not indicate evolution of physical sizes.
\end{abstract}

Key words: cosmology: observations - galaxies: evolution - galaxies: formation - galaxies: high-redshift

Supporting material: machine-readable tables

\section{INTRODUCTION}

Numerous surveys have shown that the star formation rates (SFRs) of galaxies evolve with redshift, from $z \sim 6$ (e.g., Hammer et al. 1997; Hopkins 2004; González et al. 2010; Bouwens et al. 2011) down to the local universe. From $z \sim 2$ to $z=0$, the average stellar mass of galaxies that are actively star forming, along with their SFRs, have decreased steadily (e.g., Searle et al. 1973; Cowie et al. 1996; Heavens et al. 2004; Noeske et al. 2007; Lara-López et al. 2010; Whitaker et al. 2012; Pirzkal et al. 2013). While considerable effort has been expended on studying star-forming galaxies either in the local universe or above $z \sim 1$, relatively few surveys have focused on the $\sim 7 \mathrm{Gyr}$ between the two epochs (e.g., Wolf et al. 2005; Jogee et al. 2009; Robaina et al. 2010; Lotz et al. 2011).

One SFR indicator that is particularly useful in this range is the [O II] emission doublet at $\lambda 3727$ (e.g., Kennicutt 1998; Kewley et al. 2004; Kennicutt \& Evans 2012), which is collisionally excited by the ionized electrons of $\mathrm{H}$ II regions. At $z \lesssim 1$, [O II] is easier to detect than the rest-frame UV, while being less sensitive to the effects of time-averaging of the SFR. It is, however, less straightforward to use, as its strength can be dependent on metallicity and the ionization parameter.

As part of the pilot survey for the Hobby-Eberly Telescope Dark Energy Experiment (HETDEX), Adams et al. (2011) identified 397 emission-line galaxies over $169 \operatorname{arcmin}^{2}$ of sky. While the focus of this pathfinding study was the identification of Ly $\alpha$ emitters (LAEs) at $1.9<z<3.6$ (Hill et al. 2008; Blanc

\footnotetext{
5 Institute for Gravitation and the Cosmos, The Pennsylvania State University, University Park, PA 16802, USA.
}

et al. 2011), the observations also detected a large number of lower redshift [O II] emitting galaxies. This resulted in spectra for a well-defined, emission-line flux limited sample of 284 [O II] emitters between $0<z<0.57$. While a number of SFR indicators have been previously used in this redshift range (Kennicutt 1998; Pettini et al. 2001; Hopkins 2004), this sample provides a unique resource for connecting low- and high- $z$ observations with a single, consistent SFR indicator. Ciardullo et al. (2013) has examined the [O II]-based SFRs for these galaxies and concluded that, although the scatter between the UV and [O II] SFR estimators is significant ( $\sigma \sim 0.3)$, there is no systematic offset between the techniques.

The HETDEX survey will shortly be yielding data for an emission-line selected sample of $\sim 10^{6}$ [O II] emitting galaxies in the redshift range $0<z<0.5$, with wavelength coverage from $3500 \AA$ to $5500 \AA$. In this paper, we present a pathfinding analysis of the physical and morphological properties of the [O II] emitting galaxies in the HETDEX Pilot Survey (HPS). In Section 2, we summarize this survey, and the techniques used to identify the 284 [O II] line emitters in the sample. In Section 3, we model the spectral energy distributions (SEDs) of these galaxies to produce estimates of their stellar mass, internal reddening, and SFR. In Section 4, we discuss the evolution of the SFRs in the last $\sim 5$ Gyr of cosmic time, and how the relationship between stellar mass and SFR changes over our survey epoch. In Section 5, we compare the [O II] equivalent widths of our galaxies to their stellar mass, and show that the star-forming main sequence is reflected via an anti-correlation between these two parameters. In Sections 6 and 7, we discuss the morphology and size distribution of the HPS [O II] galaxies. We show that out to $z \lesssim 0.5$, there is no evidence for significant size evolution or merger-driven starbursts. 
Throughout this work, a flat $\Lambda \mathrm{CDM}$ cosmology is used with $\Omega_{m}=0.3, \Omega_{\Lambda}=0.7$, and $H_{0}=70 \mathrm{~km} \mathrm{~s}^{-1} \mathrm{Mpc}^{-1}$ (Komatsu et al. 2011).

\section{THE HETDEX PILOT SURVEY DATA}

The data used here were taken as part of the HETDEX pilot survey (HPS; Adams et al. 2011). The survey focused on four different regions of the sky, coincident with the fields of the Cosmological Evolution Survey (COSMOS; Scoville et al. 2007), the Great Observatories Origins Deep Survey North (GOODS-N; Giavalisco et al. 2004), the Munich NearIR Cluster Survey (MUNICS; Drory et al. 2001), and the $X M M$ Large-Scale Structure survey (XMM-LSS; Pierre et al. 2004). HPS utilized the fiber-fed George and Cynthia Mitchell Spectrograph mounted on the Harlan J. Smith $2.7 \mathrm{~m}$ telescope at the McDonald Observatory. This spectrograph is a prototype for the Visible Integral-field Replicable Unit Spectrograph (VIRUS) that will eventually be used for the full HETDEX survey (Hill et al. 2008). The instrument contains 246 4".2 diameter fibers, which produced spectra from 3500 to $5800 \AA$ at a $5 \AA$ FWHM spectral resolution. For full survey details, see Adams et al. (2011), and references therein.

HPS was a blind integral field spectroscopic survey that searched for objects with emission lines within each exposure. When an emission line was found, its optical counterpart was identified in the ancillary images of COSMOS, GOODS-N, $X M M$-LSS, and MUNICS using a Bayesian matching scheme. As confirmation of the validity of the counterpart identification, for the 200 objects in the GOODS-N and COSMOS fields, we compared the redshifts obtained from the HPS spectra to spectroscopic or photometric redshifts of the counterparts obtained from the literature. In almost all cases there was complete agreement, thus confirming the identification process. Equivalent widths were estimated by comparing the HPS line flux to the flux density of the photometric continuum. The emission lines and redshifts were then identified using either other lines in the spectrum, or, since most objects possessed only a single line, an equivalent width cut. If only a single line is present in the spectrum, it is by necessity either [O II] or Ly $\alpha$ because given the spectral range of HPS, only those two emission lines would present as a single line (Adams et al. 2011). For example, if the emission line was [O III], other lines such as $\mathrm{H} \beta$ and probably [O II] would also be present.

Objects with only a single emission line detection could be either an [O II] galaxy with $z<0.57$ or an LAE with $1.9<z<3.8$, as described in Adams et al. (2011). We apply an equivalent width $(\mathrm{EW})$ cut of a rest-frame $\mathrm{EW}>20 \AA$ to identify and reject a higher redshift range of LAEs. A restframe EW > $20 \AA$ is typical for narrowband surveys of LAEs (e.g., Gronwall et al. 2007; Ciardullo et al. 2012). This restframe EW translates to an observed-frame EW > 58 to $96 \AA$ for $1.9<z<3.8$ LAEs. To confirm that our remaining galaxies are indeed lower redshift [O II] emitters and not higher redshift Ly $\alpha$ interlopers, we applied the objects' spectral energy distribution: high-redshift LAEs have no flux in the ultraviolet, as this light is shortward of the Lyman break at $912 \AA$ in the rest frame.

We are confident that the observed-frame $58 \AA \mathrm{EW}$ cut to reject LAEs does not remove a significant high-EW tail of our [O II] emitters: Hogg et al. (1998) found that $2 \%$ of [O II] emitters have $\mathrm{EW}_{\text {rest }}>60 \AA$; our sample has a median redshift of $z=0.37$, where a $58 \AA$ observed-frame EW translates to $\mathrm{EW}_{\text {rest }}$ of $42 \AA$. Considering that the [O II] distribution of EW e-folds at $20 \AA$ for the median redshift of the sample Ciardullo et al. (2013), there will be very few, if any, [O II] emitters that are close to the Hogg et al. limit.

A total of 284 [O II] emitting galaxies were identified with redshifts between $0.078<z<0.563$. Of the pointings of the survey, $90 \%$ reached a monochromatic flux limit of $1.0 \times 10^{-16} \mathrm{erg} \mathrm{cm}^{2} \mathrm{~s}^{-1}$, allowing us to recover $95 \%$ of all objects with observer-frame equivalent widths greater than $5 \AA$ (Adams et al. 2011; Ciardullo et al. 2013). The survey volume probed for the [O II] emitters is $4.24 \times 10^{4} \mathrm{Mpc}^{3}$ (Adams et al. 2011).

Of the 284 [O II] emitters, $30(\sim 10 \%)$ have X-ray counterparts from surveys by Chandra and XMM-Newton (Adams et al. 2011). This is not surprising considering the depth of the X-ray observations, particularly in the GOODS-N field, where many of the X-ray emitters were located. Ciardullo et al. (2013) showed that for most of these sources, the X-rays are likely associated with normal star formation, but $10(\sim 3 \%$ of the sample) have $\mathrm{X}$-ray luminosities greater than $10^{40} \mathrm{erg} \mathrm{s}^{-1}$ in the $2-8 \mathrm{keV}$ band. These are likely active galactic nuclei (AGNs); subsequent analysis shows that while these objects are not necessarily the brightest or the most massive in this sample, they do exhibit anomalously greater [O II] SFRs, especially at the higher redshifts in the sample. They have therefore been excluded from our analysis. For further discussion, see Ciardullo et al. (2013) and sources therein.

\section{SED FITTING}

Physical properties of the [O II] emitters were determined by fitting the SEDs of the galaxies using the Markov Chain Monte Carlo (MCMC) code GalMC (Acquaviva et al. 2011). Standard $\chi^{2}$ minimization works best if the probability distribution of the parameters is a Gaussian, and there are few variables to be fit, as the computation time grows approximately exponentially with the number of parameters (Serra et al. 2011). Additionally, $\chi^{2}$ minimization will fail to identify double-valued solutions. MCMC is advantageous because it makes no assumption about the underlying probability distribution, and any bimodal solutions are immediately obvious because in such a case, the fit will fail to converge. See Acquaviva et al. (2011) for a detailed explanation of GalMC and the MCMC algorithm.

The SED fitting was performed using up to 11 photometric bands from various sources covering a range of wavelengths from the far-UV to the IR. Table 1 lists the data that were used to perform the fits for the four survey regions. Most of the photometry was taken from Adams et al. (2011). The UV bands were found in the GR6 catalog of GALEX (Martin et al. 2005), while the data from the Spitzer $3.6 \mu \mathrm{m}$ and $4.5 \mu \mathrm{m}$ bands were gathered from publicly available catalogs. For COSMOS, this was the S-COSMOS survey (Sanders et al. 2007), for XMMLSS it was the Spitzer Wide-area Infra-Red Extragalactic survey (SWIRE; Lonsdale et al. 2003), and for GOODS-N, the IR was part of the original survey (Giavalisco et al. 2004). Aperture and PSF effects were accounted for in the photometry by the creators of the catalogs.

The GalMC SED fitting code uses the stellar population models of Bruzual \& Charlot (2003) that were updated in 2007 to incorporate improved models of thermally pulsing stars on the asymptotic giant branch (TP-AGB; Charlot \& Bruzual 2010, private communication). A constant star formation history (SFH) was assumed, along with a Salpeter (1955) initial mass function (IMF) (with $M_{\mathrm{L}}=0.1 M_{\odot}$ and $M_{\mathrm{U}}=100 M_{\odot}$ ), and a Calzetti extinction law (Calzetti et al. 2000). Nebular emission, both in the continuum and for lines, was included in proportion 
Table 1

SED Fitting Photometric Bands

\begin{tabular}{|c|c|c|c|c|c|}
\hline Field & Filter & Telescope & Instrument & $\lambda_{c}(\AA)$ & $5 \sigma$ Limit $(\mathrm{AB})$ \\
\hline \multirow[t]{9}{*}{$X M M$-LSS } & FUV & GALEX & FUV Detector & 1528 & 24.8 \\
\hline & NUV & GALEX & NUV Detector & 2271 & 24.4 \\
\hline & $u^{*}$ & CFHT & MegaPrime/MegaCam & 3740 & 25.2 \\
\hline & $g^{\prime}$ & CFHT & MegaPrime/MegaCam & 4870 & 25.5 \\
\hline & $r^{\prime}$ & CFHT & MegaPrime/MegaCam & 6250 & 25.0 \\
\hline & $i^{\prime}$ & CFHT & MegaPrime/MegaCam & 7700 & 24.8 \\
\hline & $z^{\prime}$ & CFHT & MegaPrime/MegaCam & 8900 & 23.9 \\
\hline & Channel 1 & Spitzer & IRAC & 37440 & 23.9 \\
\hline & Channel 2 & Spizter & IRAC & 44510 & 23.3 \\
\hline \multirow[t]{6}{*}{ MUNICS } & FUV & GALEX & FUV Detector & 1528 & 24.8 \\
\hline & NUV & GALEX & NUV Detector & 2271 & 24.4 \\
\hline & $B$ & Calar Alto $3.5 \mathrm{~m}$ & LAICA & 4200 & 26.4 \\
\hline & $g^{\prime}$ & Calar Alto $3.5 \mathrm{~m}$ & LAICA & 4900 & $\sim 25.9$ \\
\hline & $i^{\prime}$ & Calar Alto $3.5 \mathrm{~m}$ & LAICA & 7700 & $\sim 24.3$ \\
\hline & $z^{\prime}$ & Calar Alto $3.5 \mathrm{~m}$ & LAICA & 9200 & $\sim 24.1$ \\
\hline \multirow[t]{11}{*}{ COSMOS } & FUV & GALEX & FUV Detector & 1528 & 24.8 \\
\hline & NUV & GALEX & NUV Detector & 2271 & 24.4 \\
\hline & $u^{*}$ & CFHT & MegaPrime/MegaCam & 3740 & 26.5 \\
\hline & $B$ & Subaru & Suprime-Cam & 4788 & 27.4 \\
\hline & $V$ & Subaru & Suprime-Cam & 5730 & 27.2 \\
\hline & $r^{\prime}$ & Subaru & Suprime-Cam & 6600 & 26.9 \\
\hline & $i^{\prime}$ & Subaru & Suprime-Cam & 7850 & 26.9 \\
\hline & $z^{\prime}$ & Subaru & Suprime-Cam & 8700 & 25.6 \\
\hline & $K$ & CFHT & WIRCam & 21400 & 23.6 \\
\hline & Channel 1 & Spitzer & IRAC & 37440 & 23.9 \\
\hline & Channel 2 & Spitzer & IRAC & 44510 & 23.3 \\
\hline \multirow[t]{11}{*}{ GOODS-N } & FUV & GALEX & FUV Detector & 1528 & 24.8 \\
\hline & NUV & GALEX & NUV Detector & 2271 & 24.4 \\
\hline & $U$ & Mayall & MOSAIC & 4065 & 27.1 \\
\hline & $B$ & Suubaru & Suprime-Cam & 4788 & 27.4 \\
\hline & $V$ & Subaru & Suprime-Cam & 5730 & 27.2 \\
\hline & $r^{\prime}$ & Subaru & Suprime-Cam & 6600 & 26.9 \\
\hline & $i^{\prime}$ & Subaru & Suprime-Cam & 7850 & 26.9 \\
\hline & $z^{\prime}$ & Subaru & Suprime-Cam & 8700 & 25.6 \\
\hline & $H+K^{\prime}$ & $\mathrm{UH} 2.2 \mathrm{~m}$ & QUIRC & 20200 & 22.1 \\
\hline & Channel 1 & Spitzer & IRAC & 37440 & 23.9 \\
\hline & Channel 2 & Spitzer & IRAC & 44510 & 23.3 \\
\hline
\end{tabular}

to the rate of $\mathrm{H}$-ionizing photons, with the relative line intensities of $\mathrm{H}, \mathrm{He}, \mathrm{C}, \mathrm{N}, \mathrm{O}$, and $\mathrm{S}$ being a function of metallicity (see Acquaviva et al. 2011 for further detail). The metallicity was fixed at solar. The Lewis \& Bridle (2002) GetDist program from the publicly available CosmoMC software was used to analyze the chains output by GalMC and test for convergence via the Gelman \& Rubin (1992) $R$ statistic. The criterion employed here is $R-1<0.2$, as prescribed by Acquaviva et al. (2011). Additionally, seven objects with fewer than five photometric bands were excluded from the sample in order to ensure robust SED fits. The parameters obtained from the SED fitting were stellar mass, age since the onset of star formation, and reddening.

It is important to consider how much the results of the SED fits depend on the input models and parameters. Conroy (2013) states that the stellar mass is the most robust parameter that can be found with SED fitting techniques. He notes that generally, stellar mass can vary by $\sim 0.3$ dex for star-forming galaxies, depending on the SFH chosen, and by as much as $0.6 \mathrm{dex}$ in extreme cases. Acquaviva et al. (2011), however, state that when testing the robustness of GalMC, the choice of a constant star formation history does not result in significant differences from that when using exponentially increasing or decreasing SFHs. Additionally, Pirzkal et al. (2012) found that the choice of IMF and stellar population model using the MCMC SED fitting algorithm $\pi \mathrm{MC}^{2}$ resulted in parameter variation of a factor of a few. Metallicity estimates from SED fitting are uncertain at best, as stars of varying metallicities contribute to the overall metallicity of the galaxy in different ways as different times (Acquaviva et al. 2011). Fixing the metallicity at solar can affect the derived ages by up to $0.5 \mathrm{dex}$, but, more importantly, affects the stellar mass by only \pm 0.1 dex (Wuyts et al. 2009). This falls easily within the mass error estimates. Therefore, while the analysis presented here may contain some systematic differences, the trends will remain the same.

The median masses resulting from the SED fitting divided from redshift bin are given in Table 2 and the full results of the mass SED fitting are given in Table 3. A sample fit for a COSMOS [O II] emitter $(z=0.32)$ is given in Figure 1 . Figure 2 shows the two-dimensional confidence contours of the various parameters output by the SED fitting algorithm for the same galaxy. For the purposes of this work, the stellar mass $\left(M_{*}\right)$ is the important parameter. The stellar mass distribution of the HPS [O II] emitters is shown in Figure 3, where the masses have been divided into redshift bins. As indicated by the cumulative probability distributions, the mass distribution is fairly consistent across the entire redshift range, demonstrating the ability of an emission-line selected survey to detect low-mass galaxies at higher redshifts. 


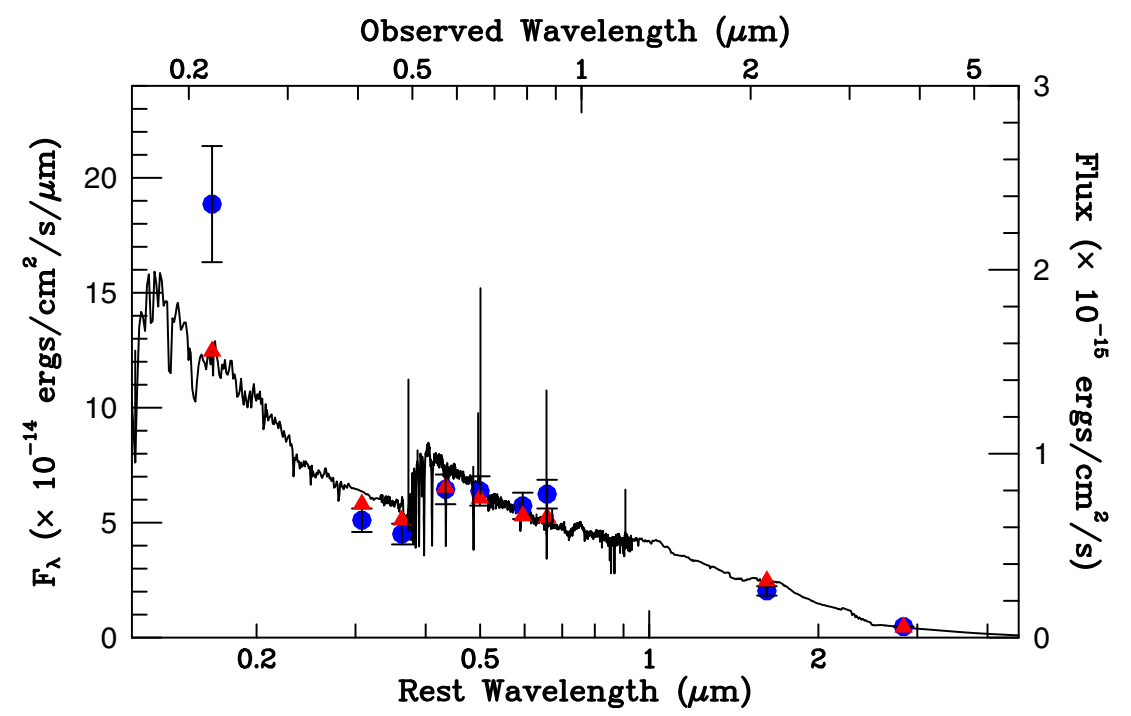

Figure 1. Typical SED fit plotted with the photometry of an [O II ] emitter $(z=0.32)$ from the COSMOS field. The blue circles are the observed flux densities, the red triangles are the fitted SED flux densities, and the black line is the SED fit.

Table 2

Median Logarithmic Galaxy Parameters by Redshift Bin

\begin{tabular}{lccc}
\hline \hline Redshift & $\begin{array}{c}\text { Mass } \\
\left(M_{*} / M_{\odot}\right)\end{array}$ & $\begin{array}{c}\text { SFR } \\
\left(M_{*} / M_{\odot} \mathrm{yr}^{-1}\right)\end{array}$ & $\begin{array}{c}\text { sSFR } \\
\left(\mathrm{yr}^{-1}\right)\end{array}$ \\
\hline $0<z<0.35$ & $9.13_{8.29}^{9.96}$ & $0.002_{-0.61}^{0.60}$ & $-9.13_{-9.91}^{-8.29}$ \\
$0.35<z<0.45$ & $9.37_{8.64}^{10.17}$ & $0.53_{0.10}^{0.92}$ & $-8.9_{-9.55}^{-8.10}$ \\
$0.45<z<0.57$ & $9.11_{8.63}^{10.22}$ & $0.70_{0.15}^{1.24}$ & $-8.56_{-9.35}^{-7.93}$ \\
Total & $9.20_{8.45}^{10.08}$ & $0.38_{-0.11}^{0.98}$ & $-8.95_{-9.66}^{-8.10}$ \\
\hline
\end{tabular}

Note. The upper and lower bounds correspond to the 84th and 16th percentiles of the distributions.

\section{INTERNAL EXTINCTION}

Accurate determination of the internal extinction in each galaxy is necessary to get reliable measurements of its [O II]based SFR. Ideally, the extinction can be calculated directly from the Balmer decrement. Unfortunately, the HPS spectral range does not include $\mathrm{H} \alpha$, and the wavelength baseline between $\mathrm{H} \beta$ and the other (weaker) Balmer lines is insufficient for our purpose. We therefore must rely on stellar-based reddening estimates; specifically, the rest-frame UV continuum slope $\beta$. Between $1250 \AA$ and $\sim 2800 \AA$, the intrinsic slope of a starforming galaxy's continuum can be well fit via a power law where $f_{\lambda} \propto \lambda^{\beta}$. Using the GALEX far-UV (FUV) and near-UV (NUV) bands, and in some cases the $u^{*}$ or $U$ (depending on the field) we calculated $\beta$ for 215 galaxies in the sample. Any flattening of this relation is most likely due to extinction, with the relationship between $\beta$ and dust extinction (Meurer et al. 1999) given by

$$
A_{1600}=4.43+1.99 \beta \text {. }
$$

This assumes an intrinsic UV spectral slope of $\beta_{0}=-2.23$, consistent with a constantly star-forming population. The reddening is then simply

$$
E_{s}(B-V)=A_{1600} / k_{1600}
$$

where $E_{s}(B-V)$ is the color excess of the stellar continuum and $k_{1600}=9.97$ is the reddening curve given by Calzetti et al. (2000).

The other 69 galaxies lacked sufficient photometry in the UV for their redshifts to do a robust UV slope calculation. In this
Table 3

Physical Parameters

\begin{tabular}{lrrrc}
\hline \hline HPS ID & $\log \left(M_{*} / M_{\odot}\right)$ & $\begin{array}{c}\log (\mathrm{SFR}) \\
\left(M_{\odot} \mathrm{yr}^{-1}\right)\end{array}$ & $E(B-V)$ & $z^{\mathrm{a}}$ \\
\hline 1 & & -0.1026 & 0.037 & 0.400 \\
2 & 9.566 & 0.2954 & 0.210 & 0.462 \\
7 & 9.708 & 0.7933 & 0.243 & 0.385 \\
8 & 8.715 & 0.2098 & 0.003 & 0.562 \\
9 & 9.966 & 0.3534 & 0.204 & 0.466 \\
10 & 8.419 & 0.3602 & 0.224 & 0.29 \\
12 & 8.352 & -0.1480 & 0.279 & 0.18 \\
14 & 10.022 & 0.4956 & 0.216 & 0.305 \\
15 & 8.235 & 0.6050 & 0.300 & 0.320 \\
16 & 8.834 & -0.3279 & 0.089 & 0.315 \\
19 & 9.142 & 1.7597 & 0.477 & 0.463 \\
20 & 10.847 & 0.5165 & 0.116 & 0.553 \\
21 & 9.143 & 0.0955 & 0.185 & 0.462 \\
23 & 9.583 & -0.4166 & 0.151 & 0.218 \\
24 & 9.389 & -0.6146 & 0.088 & 0.232 \\
26 & 8.082 & 1.5067 & 0.409 & 0.432 \\
29 & 9.438 & 0.4920 & 0.491 & 0.160 \\
31 & 9.298 & 0.6241 & 0.221 & 0.458 \\
32 & 8.955 & 0.9834 & 0.515 & 0.462 \\
33 & 9.923 & 0.2279 & 0.042 & 0.465 \\
\hline
\end{tabular}

Notes. ${ }^{a}$ Redshifts as reported in Adams et al. (2011).

(This table is available in its entirety in machine-readable form.)

case, we employed the mass-extinction relationship of Garn \& Best (2010). Using Data Release 7 of the Sloan Digital Sky Survey (SDSS), they modeled the dependency of extinction on mass as

$$
A_{H \alpha}=0.91+0.77 X+0.11 X^{2}-0.09 X^{3}
$$

where $X=\log \left(M_{*} / 10^{10} M_{\odot}\right)$. Note that since this relation is a cubic, it is only valid over a specific stellar mass range; for the 13 galaxies below $10^{8.5} M_{\odot}$ for which we do not have UV slope measurements, we assume an $E(B-V)$ set to the minimum reddening defined by the relation in Equation (3). We then employ Equation (2) to determine $E(B-V)$ using the appropriate reddening curve. This relation between stellar mass 

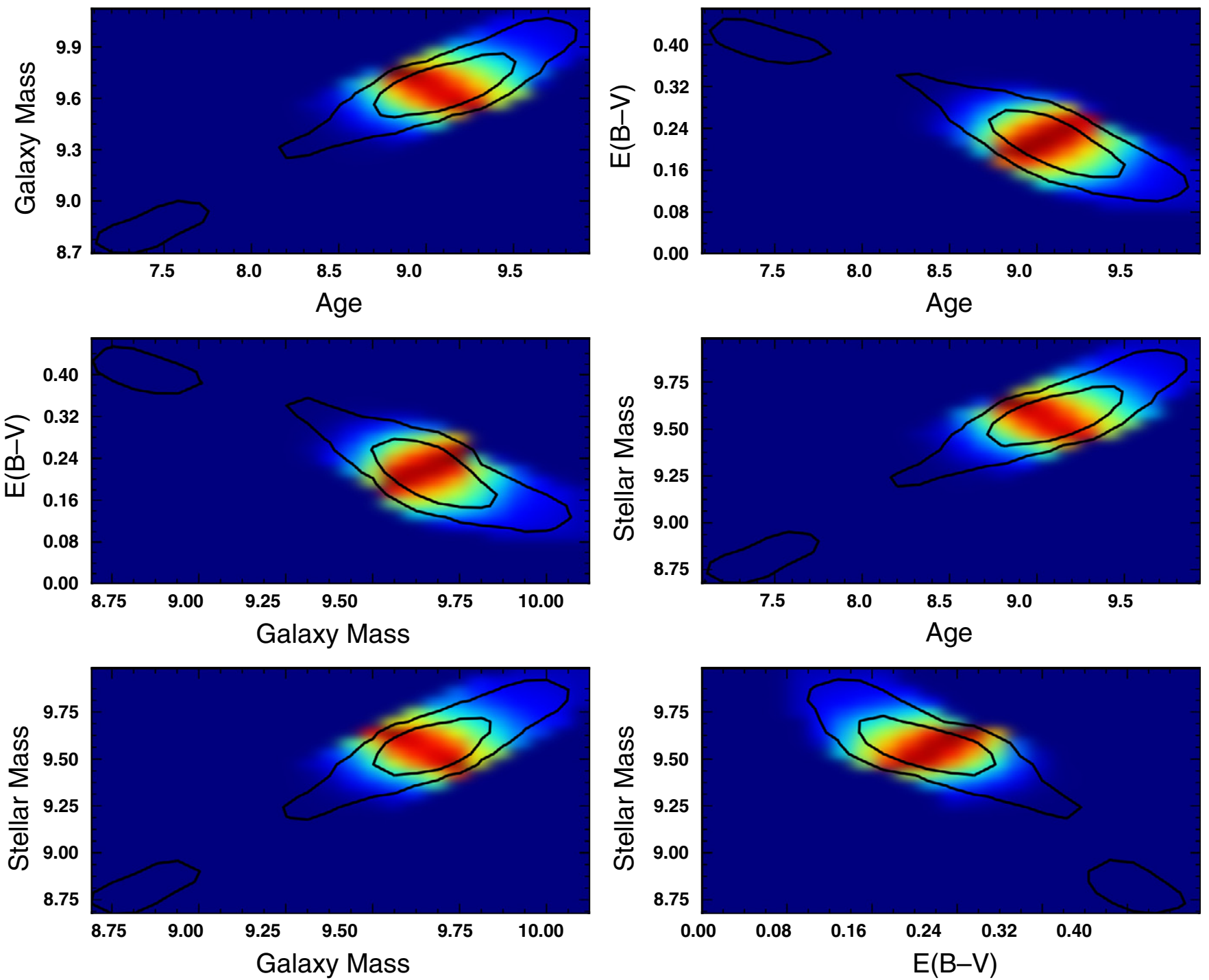

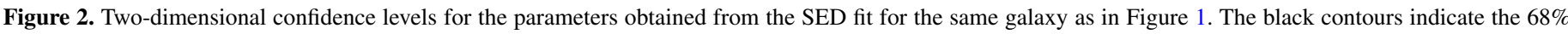

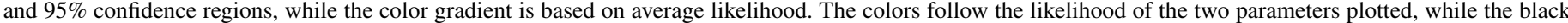

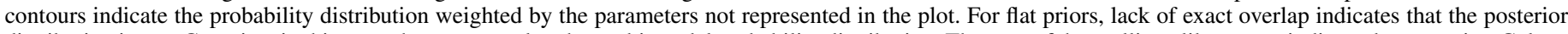

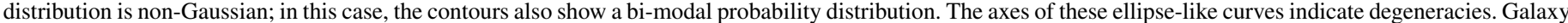

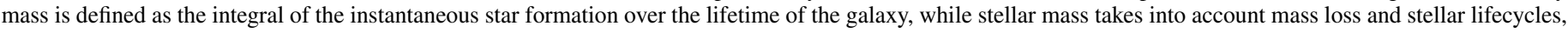
and is the parameter used throughout this analysis.

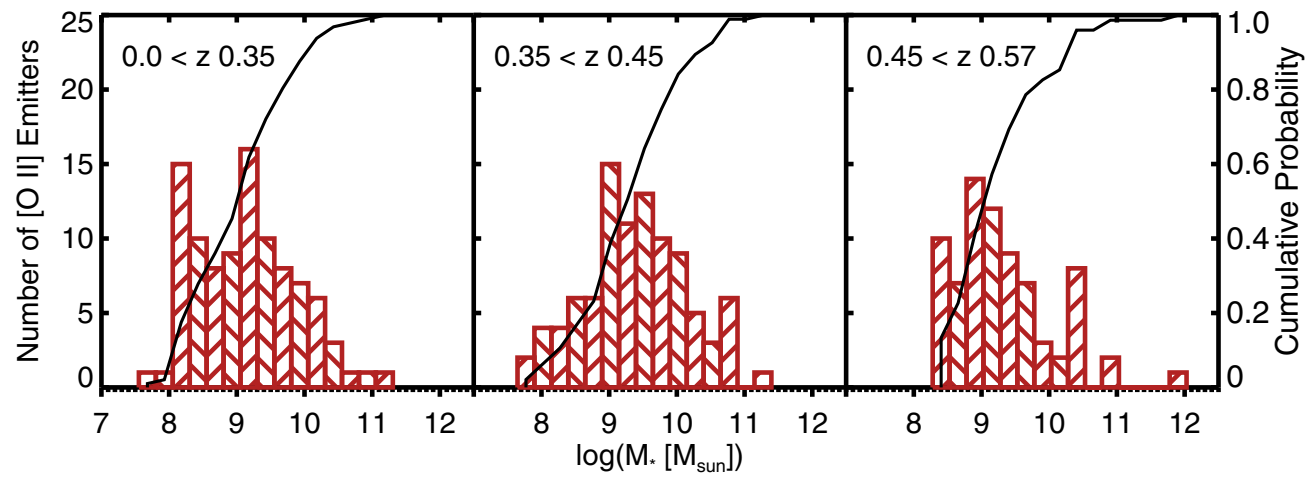

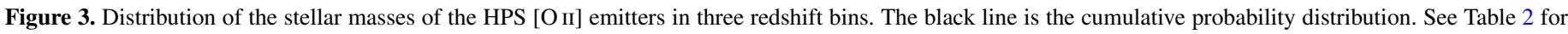
the median masses in each redshift bin. 


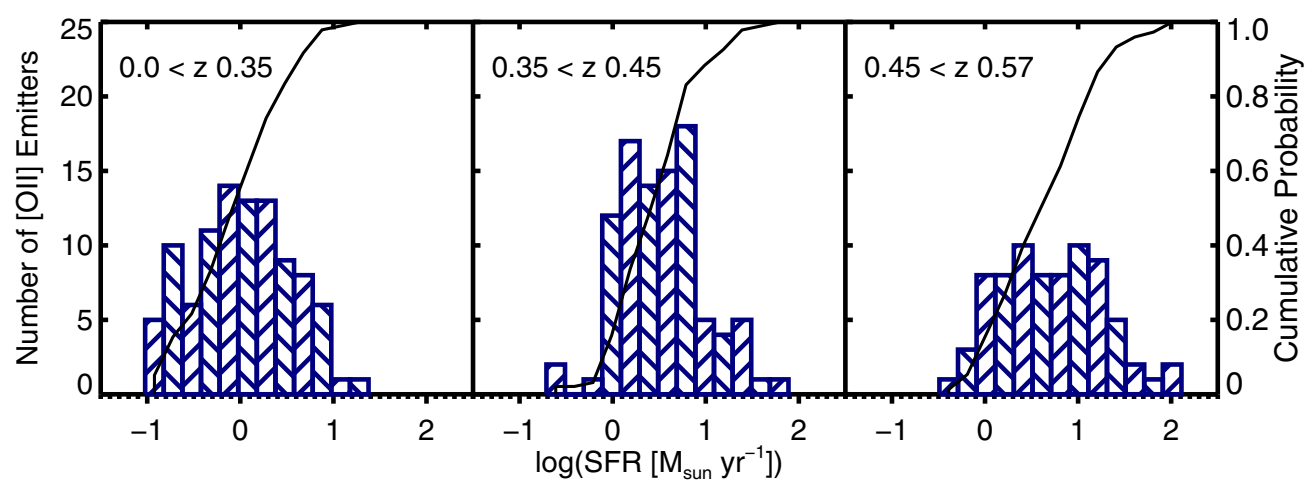

Figure 4. Distribution of the HPS [O II] SFRs calculated via their [O II] line luminosities in three redshift bins. The black line is the cumulative probability distribution. See Table 2 for the median SFRs in each redshift bin. The shifting distribution shows a clear evolution of the high star formation end of this function.

and dust has been shown to be valid out to $z \sim 1.6$ (Zahid et al. 2014).

Finally, the entire sample was corrected for extinction using the Calzetti et al. (2000) extinction law

$$
F_{i}(\lambda)=F_{o}(\lambda) 10^{0.4 E_{s}(B-V) k(\lambda)} .
$$

Note that $E_{s}(B-V)$ is related to the color excess of the nebular emission lines, such as [O II], by

$$
E_{s}(B-V)=0.44 E(B-V)
$$

(Calzetti et al. 2000).

\section{SFRs}

There has been extensive discussion in the literature about the "main sequence" of star-forming galaxies, i.e., the correlation between a galaxy's SFR and its stellar mass (e.g., Noeske et al. 2007). Both the slope and, more strongly, the normalization of the sequence has also been shown to evolve with redshift (Whitaker et al. 2012).

The SFRs for the HPS [O II] emitters were calculated using the $[\mathrm{O}$ II] $\lambda 3727$ Kewley et al. (2004) relation

$$
\operatorname{SFR}([\mathrm{O} \text { II }])\left(M_{\odot} \mathrm{yr}^{-1}\right)=(6.53 \pm 1.65) \times 10^{-42} L([\mathrm{O} \text { II }])\left(\mathrm{erg} \mathrm{s}^{-1}\right)
$$

where $L([\mathrm{O} \mathrm{II}])$ is the galaxy's [O II] luminosity. Note that this is a mean calibration since the wavelength range for HPS does not include the $\mathrm{H} \beta$ or [O III] $\lambda 5007$ emission lines that would allow fine-tuning for the effects of variations in oxygen abundance and ionization states.

The distribution of the SFRs of the galaxies in our sample is given in Figure 4. The median SFRs of the [O II] emitters are given in Table 2. The evolution of star formation with redshift for the [O II] emitters is presented in Figure 5, partially reflecting the changing luminosity limit of the survey. However, after taking this into account, there is a clear relationship between the SFR of the galaxies and their redshifts, with the star formation tending to decrease at lower redshifts. Figure 6 compares the SFR and mass of the HPS [O II] emitters.

Previously, Noeske et al. (2007) and Pirzkal et al. (2013) have examined the main sequence of star-forming galaxies at slightly higher redshifts. Noeske et al. (2007) analyzed a magnitudelimited set of star-forming galaxies from the Extended Groth Strip (AEGIS), probing galaxies of high mass within a redshift range of $0.2<z<1.1$ using Spitzer (MIPS) $24 \mu \mathrm{m}$ imaging as well as $\mathrm{H} \alpha, \mathrm{H} \beta$, and [O II] emission lines. Similarly, Pirzkal et al. (2013) studied emission-line galaxies (ELGs) from the GOODS-N and -S fields as part of the Probing Evolution And Reionization Spectroscopically (PEARS) survey. This slitless grism survey identified $\mathrm{H} \alpha$, [O III], and [O II] in the redshift ranges of $0<z<0.5,0.1<z<0.9$, and $0.5<z<1.5$, respectively. It should be noted that in their determination of stellar mass via SED fitting, Pirzkal et al. assumed a constant $\mathrm{SFH}$, as was assumed in this survey, while Noeske et al. used an exponentially decreasing SFH. Figure 7 compares these data to our own measurements to show the progression of the star formation sequence. The addition of the HPS [O II] emitters extends the galaxy star-forming main sequence of galaxies down to low redshifts, allowing for comparison of SFRs over a broad range of masses and redshifts. As with the other galaxies, the [O II] emitters show an evolution of SFR with redshift that is consistent with a galactic SFR evolution along the main sequence of star-forming galaxies.

To determine the intrinsic slope and scatter of the main sequence, we must make mass cuts to account for incompleteness in the SFRs. This is an expected characteristic of an emissionline selected sample: while the underlying distribution may have a given slope, if a sample is limited by the SFRs of the galaxies, the apparent shape of the SFR- $M_{*}$ relation will be skewed flatter than that for an unbiased sample. Conversely, a mass-selected sample, such as the AEGIS survey, will produce a steeper slope. To address this, we performed mass cuts in the four redshift bins represented in Figure 6 by implementing an iterative algorithm using the slope and scatter of distribution to determine where the cuts should be made. For $z<0.2$, we made no cuts, as we expect the data are essentially complete at this low redshift. For $0.2<z<0.35$, we invoked a mass cut at $10^{8.2} M_{\odot}$, for $0.35<z<0.45$ we used $10^{9.1} M_{\odot}$, and for the highest redshift bin of $0.45<z<0.57$ we used $10^{9.7} M_{\odot}$. Because this is an emission line-selected sample, and care was taken to ensure robust photometry for all objects included, after having performed the mass cuts, the resulting sample is complete in both SFR and mass in the given redshift ranges.

The dispersion in the star formation sequence of the HPS [O II] emitters is about $\sigma_{\mathrm{MS}} \sim 0.50 \mathrm{dex}$, about 1.5 times as large as some other surveys (e.g., Noeske et al. 2007; Whitaker et al. 2012). Because our observations included just the [O II] line, our SFRs do not take into account variations in abundance and ionization state. This undoubtedly exacerbates the scatter in our star-forming main sequence by as much as 0.15 dex (Lara-López et al. 2013). Yet another source of additional scatter is that we invoke Garn \& Best (2010) which can add up to $\sim 0.28$ dex 


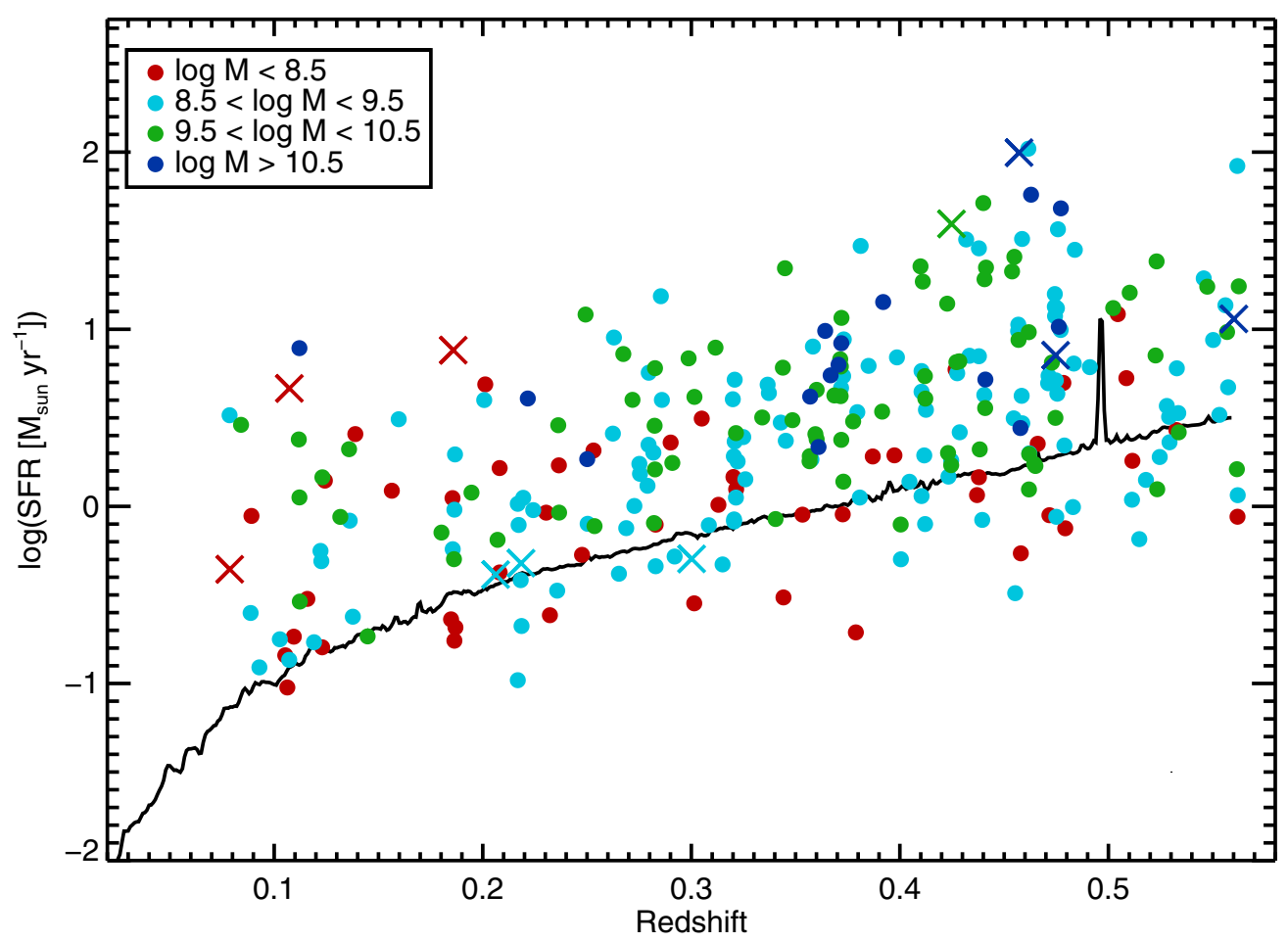

Figure 5. Evolution of the SFRs of the HPS [O II] emitters as a function of redshift, color coded by mass. The black line represents the detection limit as a function of redshift, where $80 \%$ of the survey frames have $5 \sigma$ detection limits brighter than this threshold. The prominent [O I] airglow feature at $5577 \AA$ at $z \sim 0.5$ is clearly visible. The crosses are possible AGN candidates due to their bright X-ray emission. The representative SFR error bar is shown.

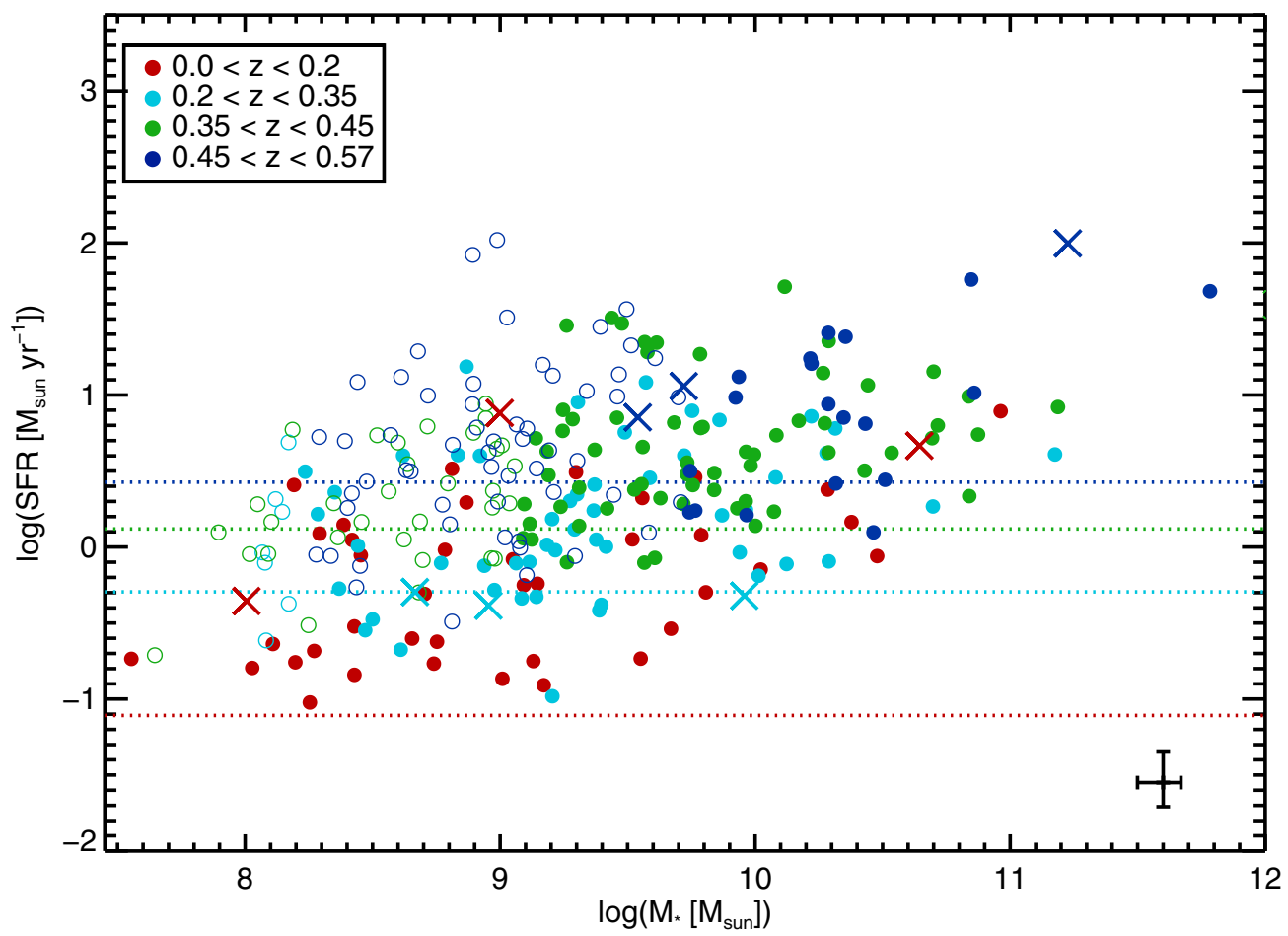

Figure 6. SFR vs. stellar mass for the HPS [O II] emitters. The crosses are possible AGN candidates due to their bright X-ray emission. Representative error bars are shown. The open circles represent galaxies below the stellar masses where SFR becomes incomplete in each redshift bin.

in scatter. Finally, because our emission-line selected survey is sensitive to galaxies with lower SFRs, it is more prone to scatter in the star formation sequence, especially at lower masses. The larger $\left(\sigma_{\mathrm{MS}} \sim 0.45 \mathrm{dex}\right)$ dispersion seen in the grism data of Pirzkal et al. (2013) supports this interpretation. Additionally, Bauer et al. (2013) used $\mathrm{H} \alpha$ luminosities to determine the SFR of the $\sim 73,000$ galaxies with redshifts of $0.05<z<0.32$ in the emission-line selected Galaxy And Mass Assembly (GAMA) survey, and found significant scatter in the main sequence, up to $\sim 1$ dex in the highest mass bin.

The slope of this main sequence is somewhat shallower than comparable studies for the [O II] emitting galaxies with redshift 


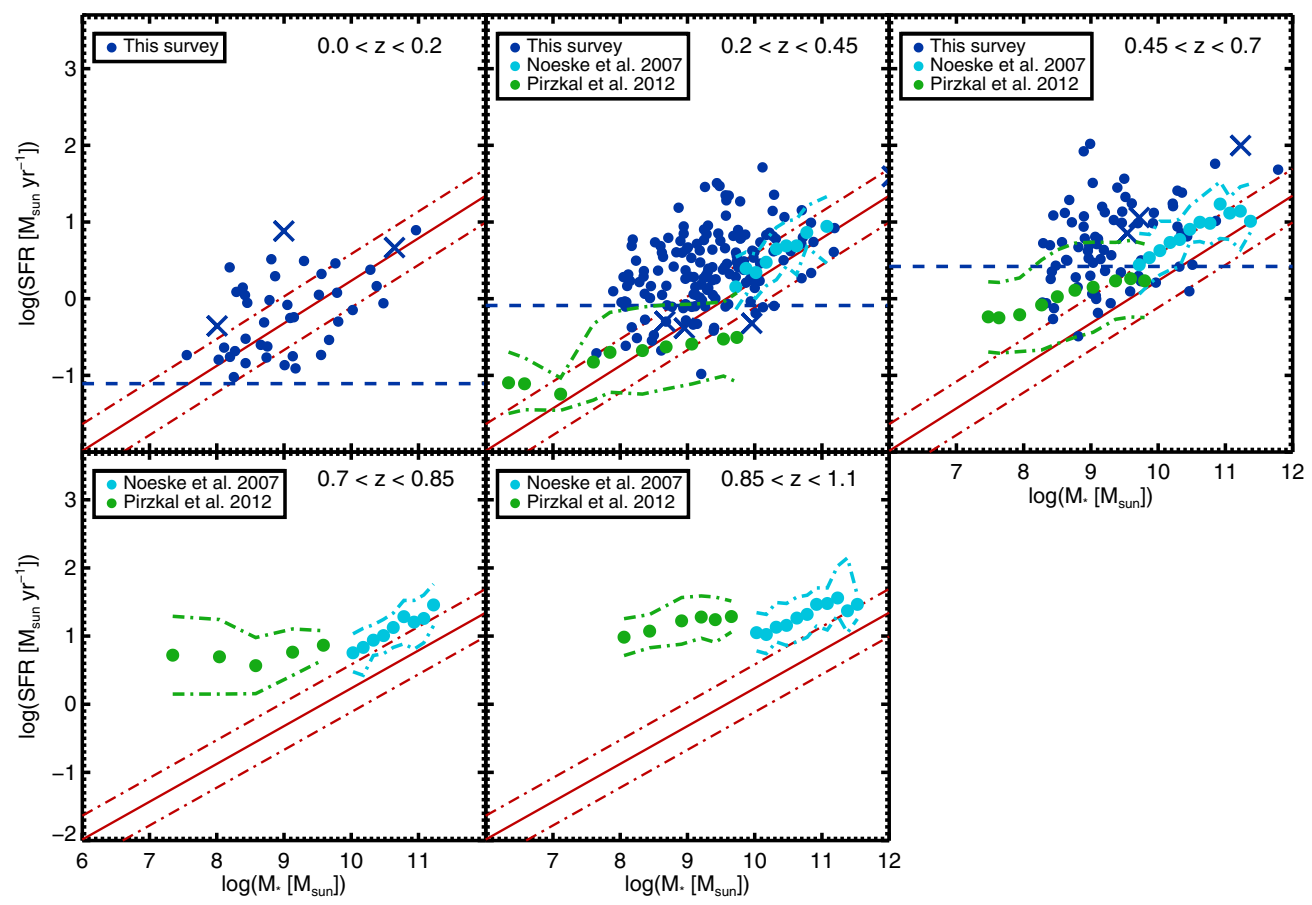

Figure 7. SFR vs. stellar mass for the HPS [O II] emitters, as well as the Noeske et al. (2007) and Pirzkal et al. (2013) median values. The darker blue circles represent the HPS galaxies and the dark blue crosses are the possible AGNs in the sample. The green circles are the Pirzkal et al. median values, and the light blue circles are the Noeske et al. median values. The light blue and green dash-dotted lines show $\pm 1 \sigma$ of the median values shown. The darker blue dashed line represents the $80 \%$ completeness limit for the middle redshift of each plot. The red solid line is taken from Lara-López et al. (2013) and denotes the $M_{*}-\mathrm{SFR}$ relation for local galaxies up to $z \sim 0.1$, and the red dash-dotted lines are $\pm 1 \sigma$ of that relation. This relation is included in the higher redshift bins only to guide the eye.

$z<0.5$. A linear fit for the HPS sample produces

$\log (\mathrm{SFR})\left(M_{\odot} \mathrm{yr}^{-1}\right)=(0.47 \pm 0.05) \log \left(M_{*} / M_{\odot}\right)-(4.10 \pm 0.49)$

over the entire mass range in this sample. Noeske et al. (2007 a) report a fit of

$\log (\mathrm{SFR})\left(M_{\odot} \mathrm{yr}^{-1}\right)=(0.67 \pm 0.08) \log \left(M_{*} / M_{\odot}\right)-(6.19 \pm 0.78)$

for $M_{*}$ between $10^{10}$ and $10^{11} M_{\odot}$ in the redshift range $0.2<$ $z<0.7$. For the local universe, data from the Sloan Digital Sky Survey-Data Release 7 (SDSS-DR7) yield

$$
\log (\mathrm{SFR})\left(M_{\odot} \mathrm{yr}^{-1}\right)=0.55 \log \left(M_{*} / M_{\odot}\right)-5.31
$$

with $\sigma=0.349$ (Lara-López et al. 2013). Because the SDSS/ GAMA survey includes galaxies with $z \leqslant 0.1$ and the AEGIS sample (Noeske et al. 2007) is in the range $z=0.2-0.7$, we would expect the HPS sample to fall in the same general category of slope and normalization. However, our galaxies have a slightly flatter slope; this could partly be due to the fact that HPS is an emission-line selected survey, meaning that the SFR$M_{*}$ parameter space being explored is slightly different than the ones detected by continuum-selected surveys.

What is more likely affecting the slope of the main sequence, however, is that, as stated previously, the [O II] SFR indicator is sensitive to metallicity, something we are not able to measure individually with the HPS data. However, if we adopt a mass-metallicity relationship, we can estimate the effect that abundance shifts have on the slope. Lara-López et al. (2013) give a mass-metallicity relationship of

$$
\begin{aligned}
12+\log (\mathrm{O} / \mathrm{H})= & -10.8297+3.6478 \log \left(M_{*} / M_{\odot}\right) \\
& -0.16706 \log \left(M_{*} / M_{\odot}\right)^{2} .
\end{aligned}
$$

This law, and the Kewley et al. (2004) relationship between SFR, [O II] luminosity, and metallicity

$$
\begin{aligned}
& \mathrm{SFR}([\mathrm{O} \mathrm{II}], Z)\left(M_{\odot} \mathrm{yr}^{-1}\right) \\
& \quad=\frac{7.9 \times 10^{-42} L([\mathrm{O} \mathrm{II}])\left(\mathrm{erg} \mathrm{s}^{-1}\right)}{(-1.75 \pm 0.25)[12+\log (\mathrm{O} / \mathrm{H})]+(16.73 \pm 2.23)}
\end{aligned}
$$

give the modified median SFR per mass bin shown in Figure 8. Including a rough estimate of the metallicity moves the higher mass galaxies to larger SFRs and the lower mass galaxies to lower SFRs, steepening the slope to

$$
\begin{aligned}
\log (\mathrm{SFR})\left(M_{\odot} \mathrm{yr}^{-1}\right)= & (0.68 \pm 0.05) \log \left(M_{*} / M_{\odot}\right) \\
& -(6.21 \pm 0.50) .
\end{aligned}
$$

This is on par with what has been found in previous studies (e.g., Noeske et al. 2007; Whitaker et al. 2012), and illustrates the importance of metallicity to the [O II] SFR indicator.

Figure 7 confirms the results of Noeske et al. (2007) and Whitaker et al. (2012) on the evolution of the main sequence, as the entire main sequence appears to shift downward with redshift. The normalization of the main sequence decreases by almost a factor of two from the highest redshift bin (median $z \sim 0.51$ ) to the lowest (median $z \sim 0.1$ ).

Another way to describe star formation in a galaxy is through its mass specific SFR (sSFR), which represents the time needed to build up the current stellar mass of the galaxy at its present day SFR (Pirzkal et al. 2013). The sSFR of galaxies has been shown at all redshifts to decrease as stellar mass increases (e.g., Bauer et al. 2005). Figure 9 shows the distribution of the [O II] emitters' sSFRs calculated via their [O II] line luminosities. The median sSFRs per redshift bin are given in Table 2. The distribution of the HPS [O II] emitters shifts to larger sSFRs as redshift increases, indicating that galaxies at higher redshifts are more 


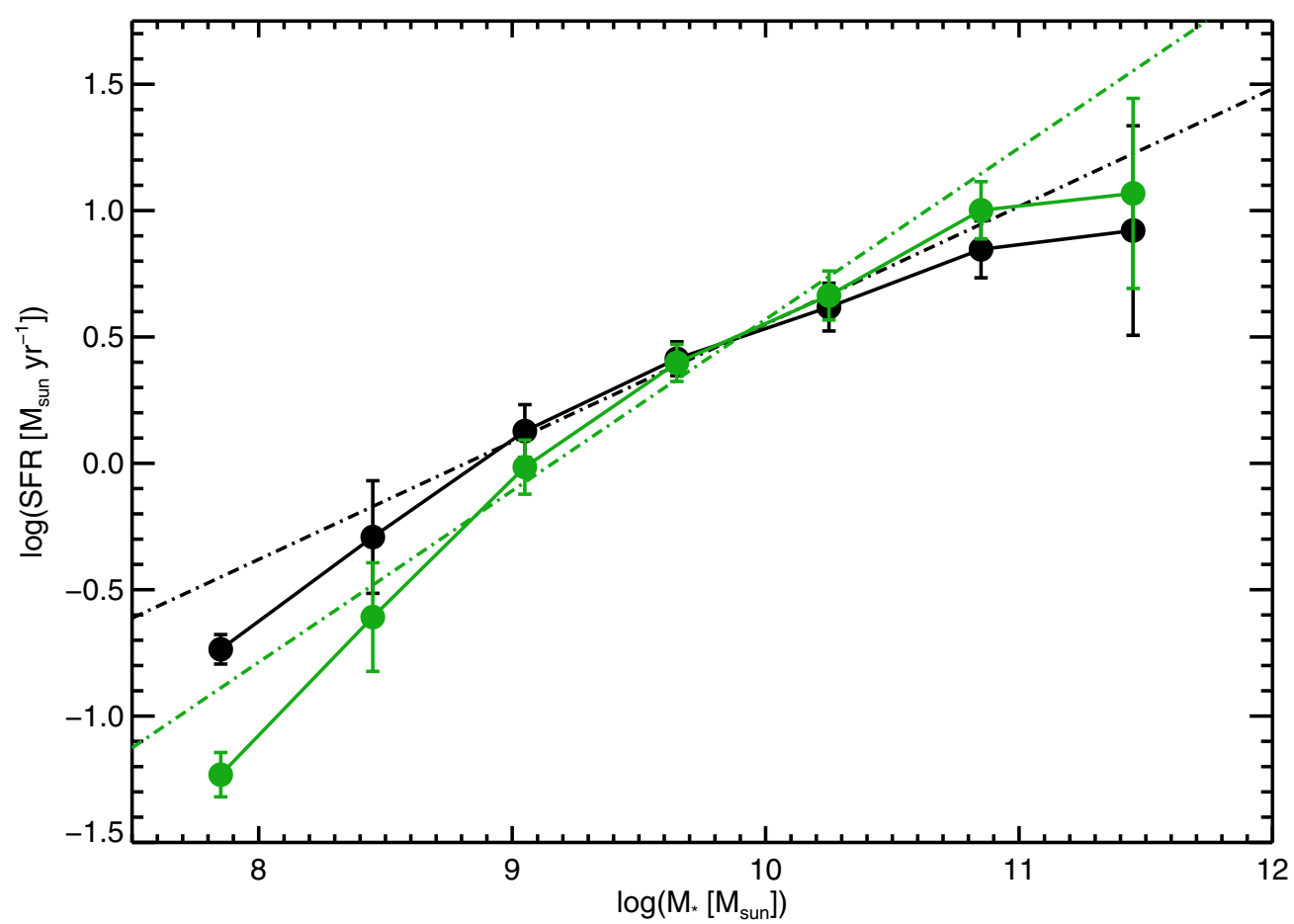

Figure 8. Median SFR vs. stellar mass for the HPS [O II] emitters. The black circles represent the median SFRs in evenly spaced mass bins, calculated without the metallicity correction to the [O II] SFR indicator. The green circles shows the median SFRs calculations that include a rough estimate of metallicity. The corresponding dash-dotted lines give the best linear fits to the data. The error bars represent the uncertainty of the medians. Inclusion of the metallicity in the SFR calculation drives the sequence to a steeper slope.

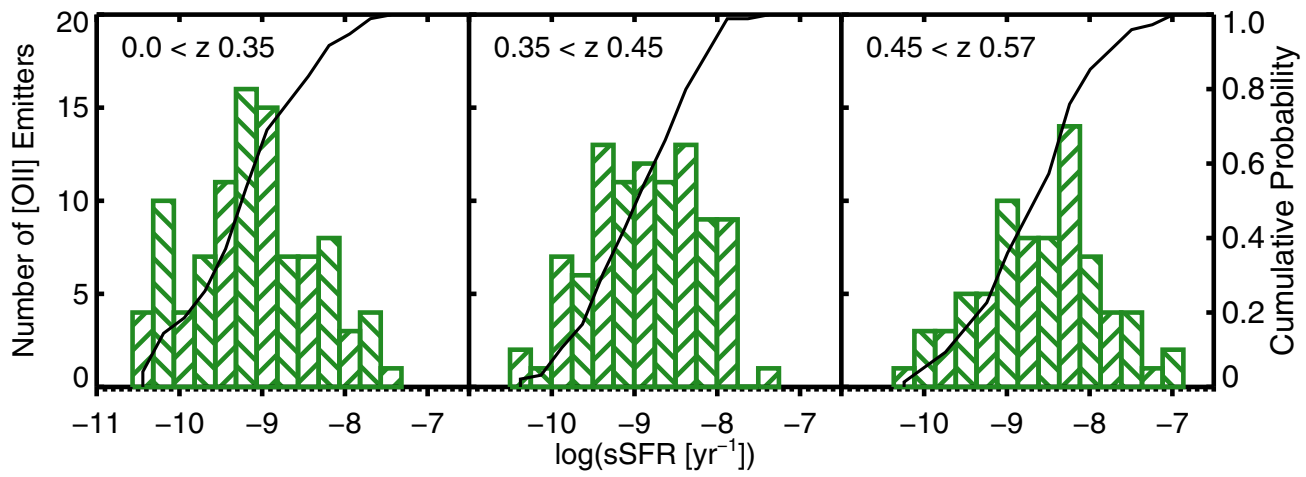

Figure 9. Distribution of the HPS [O II] emitter sSFRs calculated via their [O II] line luminosities. The black line is the cumulative probability distribution. See Table 2 for the median sSFRs in each redshift bin.

efficient at producing stars. Figure 10 indicates that there is a trend for the more massive galaxies to be formed at higher redshifts and over a short burst timescales (Cowie et al. 1996), but by $z \sim 0.5$, their sSFR has decreased. At that time, the lower mass galaxies have begun their (relatively) later onset of star formation, indicating that they have formed the bulk of their stars more recently but on longer evolutionary timescales. These data are consistent with the concept of downsizing as described by Cowie et al., where there is a smooth evolution downward with redshift in the masses of star-forming galaxies.

\section{EQUIVALENT WIDTHS}

Figure 11 shows the [O II] rest-frame equivalent widths (EWs) as a function of mass for the HPS galaxies. As found in other surveys (e.g., Fumagalli et al. 2012) there is a weak anticorrelation between EW and stellar mass. In the lowest mass bin, the median rest-frame equivalent width is three times greater than that in the highest mass bin. For comparison, Fumagalli et al. found a factor of five shift in the EWs of $\mathrm{H} \alpha$ between the mass bins of $10.0<\log M / M_{\odot}<10.5$, and $\log M / M_{\odot}>11.0$. The two measurements are not directly comparable, since, in addition to being in the redder range of the spectrum, the $\mathrm{H} \alpha$ data were accumulated over a larger redshift range. Nevertheless, the data do confirm the trend that emissionline equivalent widths in higher-mass galaxies are generally factors of several lower than those in lower-mass objects. Since the equivalent width of [O II] is an indicator of the relative strength of star formation (Gilbank et al. 2010; Ciardullo et al. 2013), this trend is another reflection of the star-forming galaxies main sequence.

\section{MORPHOLOGIES}

The HPS observations were conducted in regions of the sky with extensive ancillary data. In particular, in the COSMOS and 


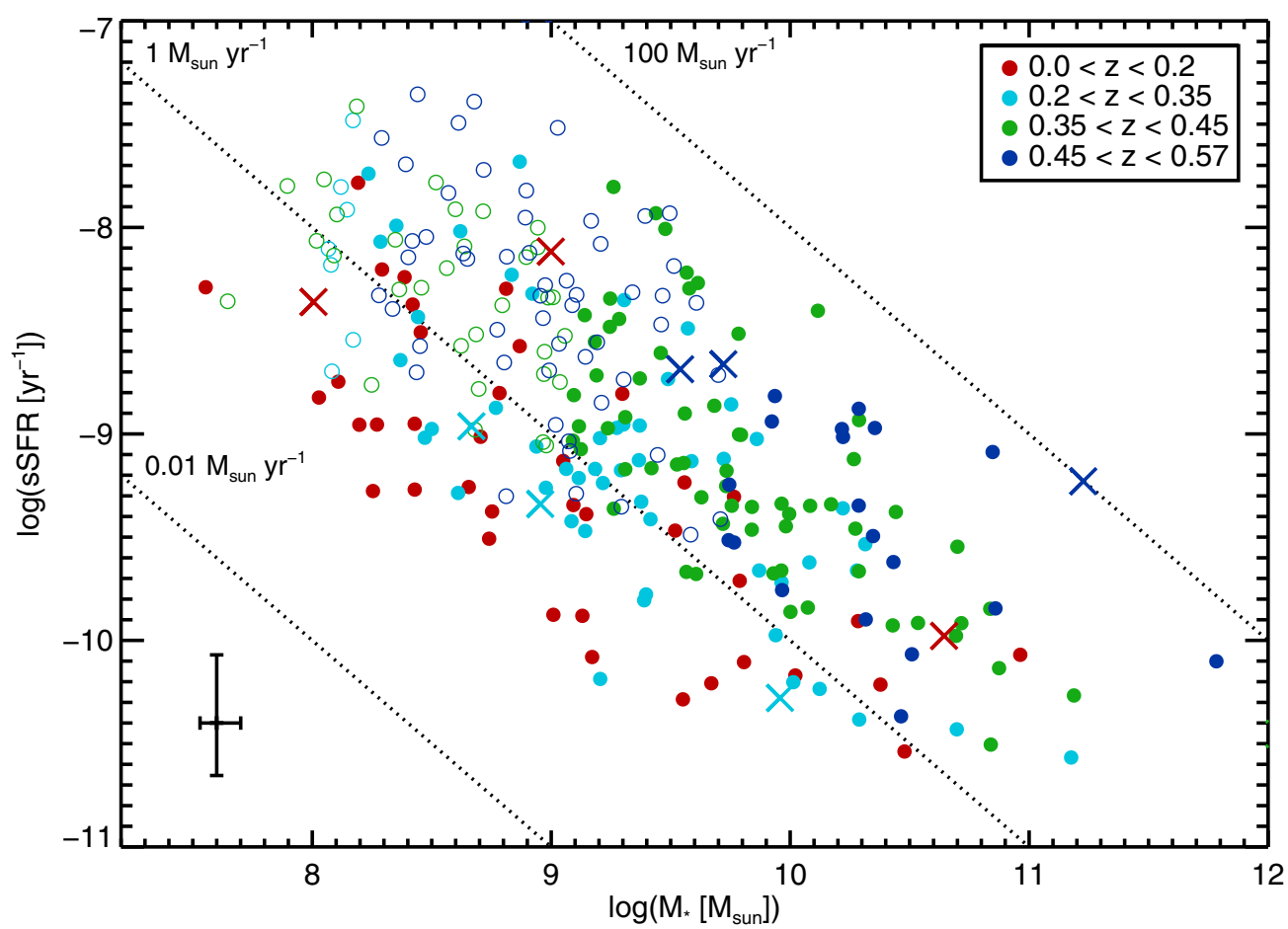

Figure 10. sSFR vs. $M_{*}$ for the HPS [O II] emitters. The diagonal black dotted lines are loci of constant star formation with values $0.01,1$, and $100 M_{\odot} \mathrm{yr}^{-1}$, from bottom to top. The crosses are possible AGN candidates due to their bright X-ray emission. Representative error bars are shown. The open circles represent galaxies below the stellar masses where SFR become incomplete in each redshift bin.

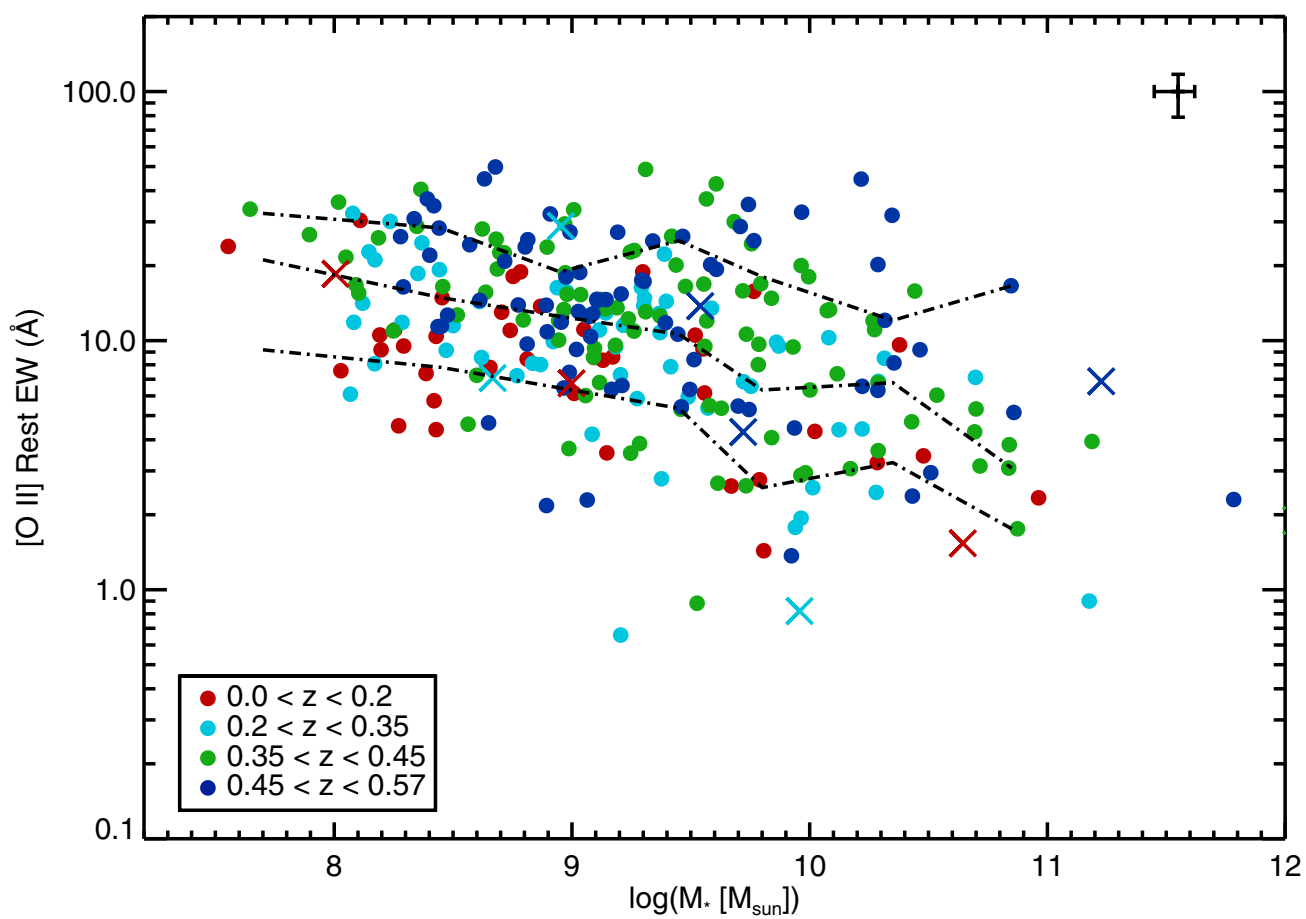

Figure 11. Rest equivalent widths of the HPS [O II] lines as a function of stellar mass. The black dash-dotted lines are, from top to bottom, the 84th, 50th, and 16th percentiles in the given mass bins. The crosses are possible AGN candidates due to their bright X-ray emission. Representative error bars are shown.

GOODS-N regions, deep imaging data is available from the Hubble Space Telescope Advanced Camera for Surveys (ACS). These data allow us to characterize the morphology and size of the HPS [O II] emitters via the Gini (G) and $M_{20}$ coefficients (Lotz et al. 2004). To do this, we chose to use data in the F814W filter, as it is the only filter common to both the COSMOS
(Scoville et al. 2007) and GOODS-N (Giavalisco et al. 2004) programs.

The $G$ coefficient represents the distribution of the flux values over the galaxy's pixels, and is thus similar to the concentration parameter $C$ (e.g., Abraham et al. 1994; Bershady et al. 2000; Conselice 2003), which, loosely defined, is the ratio of light 


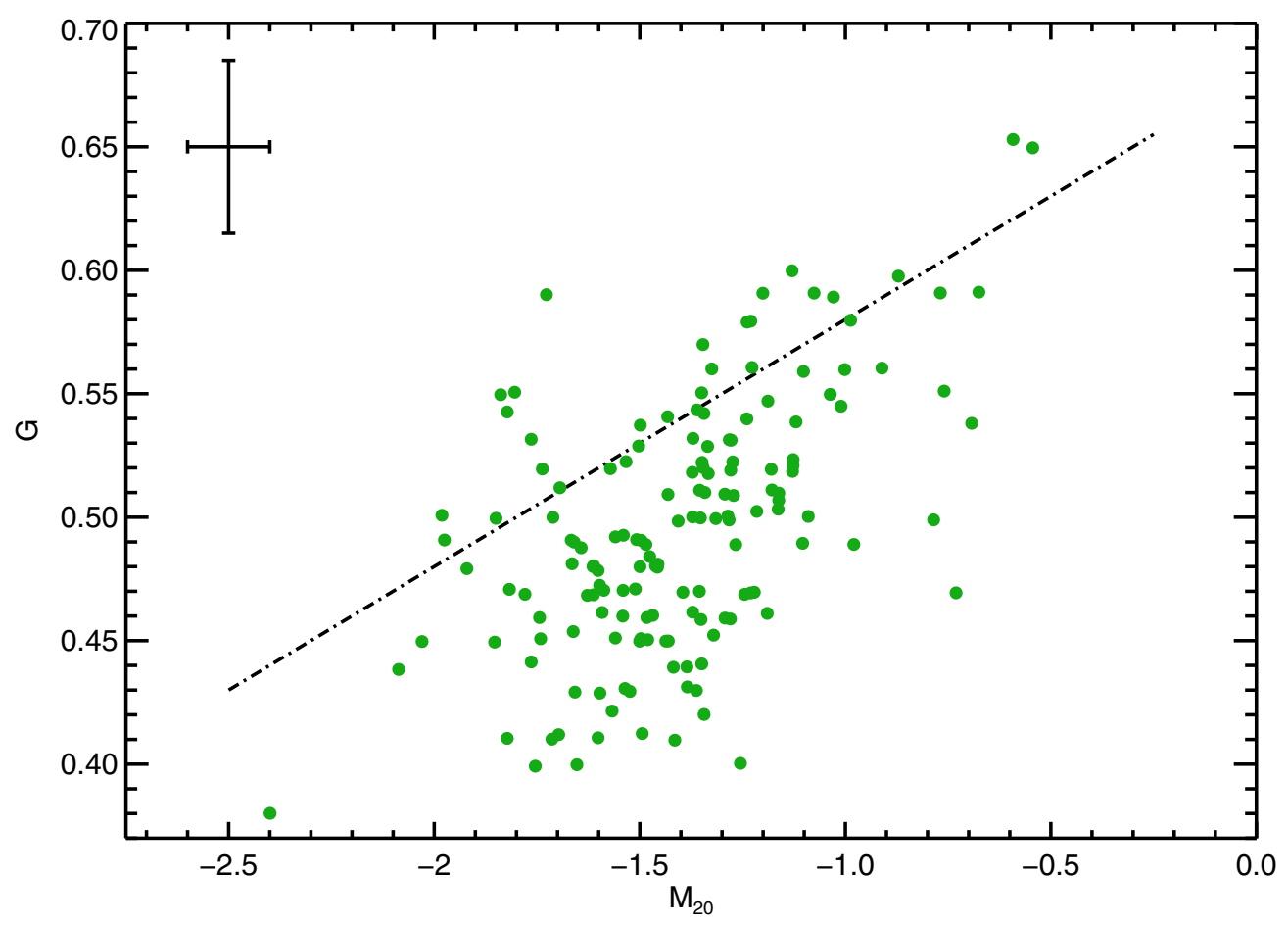

Figure 12. Morphological parameters $G$ and $M_{20}$ for the [O II] emitters in the COSMOS and GOODS-N fields. The delineation between galaxies with disturbed morphologies and normal galaxies (black line) is taken from Lotz et al. (2004) and is for reference purposes only. Representative error bars are adapted from Lotz et al. (2006) and are typical for a $\langle\mathrm{S} / \mathrm{N}\rangle=2.5$ galaxy.

between inner and outer isophotes of a galaxy. However, $C$ is dependent on the spatial distribution of the light and is unable to differentiate between galaxies with off-centered light concentrations and those with shallow light profiles. The Gini coefficient is sensitive to concentrations of bright pixels no matter where in the profile they occur, resulting in a more robust measure of concentration.

The $M_{20}$ coefficient is defined as the second-order moment of the brightest $20 \%$ of the flux of a galaxy. The second-order moment of flux $\left(M_{\mathrm{tot}}\right)$ is calculated by multiplying each pixel's flux by the squared distance to the center of the galaxy, and then summing over all the pixels:

$$
M_{\mathrm{tot}}=\sum_{i}^{n} M_{i}=\sum_{i}^{n} f_{i}\left[\left(x_{i}-x_{c}\right)^{2}+\left(y_{i}-y_{c}\right)^{2}\right]
$$

where $f_{i}$ is the pixel flux and $x_{c}, y_{c}$ is the center of the galaxy (Lotz et al. 2004). Selecting the brightest $20 \%$ of the pixels results in a parameter that is sensitive to the most important spatial distributions of the flux, such as multiple nuclei, bars, and star clusters. Therefore, $M_{20}$ probes features indicative of star formation.

Gini and $M_{20}$ are dependent on the noise and resolution of the images, and are not reliable if the signal-to-noise per pixel drops below 2 (Lotz et al. 2004). Hence, galaxies with $\langle S / N\rangle$ less than this $(\sim 15 \%$ of the sample) were excluded from our analysis. Additionally, $G$ is only reliable to within $\sim 15 \%$ at resolution scales better than $1000 \mathrm{pc}$ while $M_{20}$ begins to degrade around $500 \mathrm{pc}$. At the HPS redshift limit for [O II] detection, the $\sim 0^{\prime} .05 /$ pixel plate scale of ACS corresponds to a resolution of $\sim 330 \mathrm{pc}$. Assuming a spatial resolution no worse than $\sim 0$ !' 1 , the highest redshift in our sample corresponds to a $\sim 650 \mathrm{pc}$ resolution. Therefore, while $M_{20}$ for the very highest redshift galaxies in the sample may have some degradation above $15 \%, M_{20}$ and
Table 4

Morphological Parameters and Sizes

\begin{tabular}{llll}
\hline \hline HPS ID & $G$ & $M_{20}$ & $\begin{array}{c}R_{50} \\
(\mathrm{kpc})\end{array}$ \\
\hline 143 & & & 0.863 \\
146 & 0.56 & -1.68 & 2.179 \\
147 & 0.49 & -1.5 & 2.318 \\
149 & 0.47 & -1.47 & 1.407 \\
151 & 0.5 & -1.72 & 2.041 \\
152 & 0.51 & -1.84 & 2.834 \\
155 & 0.55 & -1.97 & 1.178 \\
158 & 0.59 & -1.97 & 0.674 \\
163 & 0.56 & -2.0 & 2.779 \\
165 & 0.49 & -1.44 & 2.227 \\
\end{tabular}

(This table is available in its entirety in machine-readable form.)

$G$ coefficients should still be for the most part reliable over the entire sample.

The morphology parameters of the COSMOS and GOODS$\mathrm{N}$ [O II] emitters were calculated using the Lotz et al. (2004) software, which uses SExtractor catalogs and segmentation maps. The distribution of these galaxies in $G-M_{20}$ space is plotted in Figure 12, and the results are tabulated in Table 4. Lotz et al. (2004) have shown that active or merging galaxies tend to lie above a certain threshold in $G-M_{20}$ parameter space. Only a fraction of the [O II] emitters in this sample fall above this threshold. Instead, the [O II] emitters fall largely within the region of nonmerging galaxies. Indeed, a cursory examination of HST/ACS images of the [O II] emitters confirms that most of these galaxies are not strongly distorted or visibly interacting.

This result is divergent from the results of Pirzkal et al. (2013), who found that a significant fraction of the emission-line 


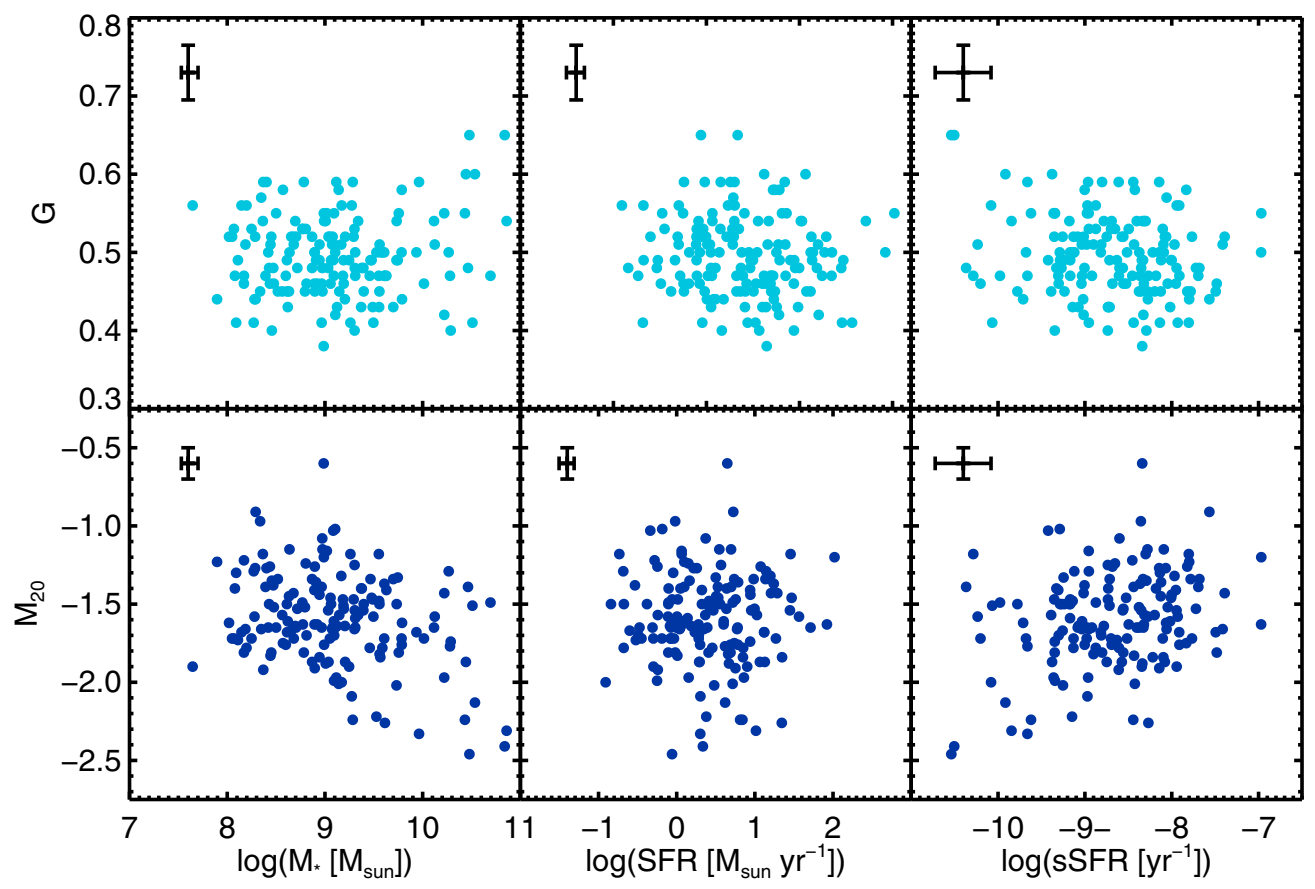

Figure 13. Morphological parameters $G$ and $M_{20}$ plotted as a function of stellar mass, SFR, and sSFR. Pearson's correlation coefficient shows almost no significant correlation between the values. Representative error bars are shown, with those for $G$ and $M_{20}$ adapted from Lotz et al. (2006), and are typical for a $\langle\mathrm{S} / \mathrm{N}\rangle=2.5$ galaxy.

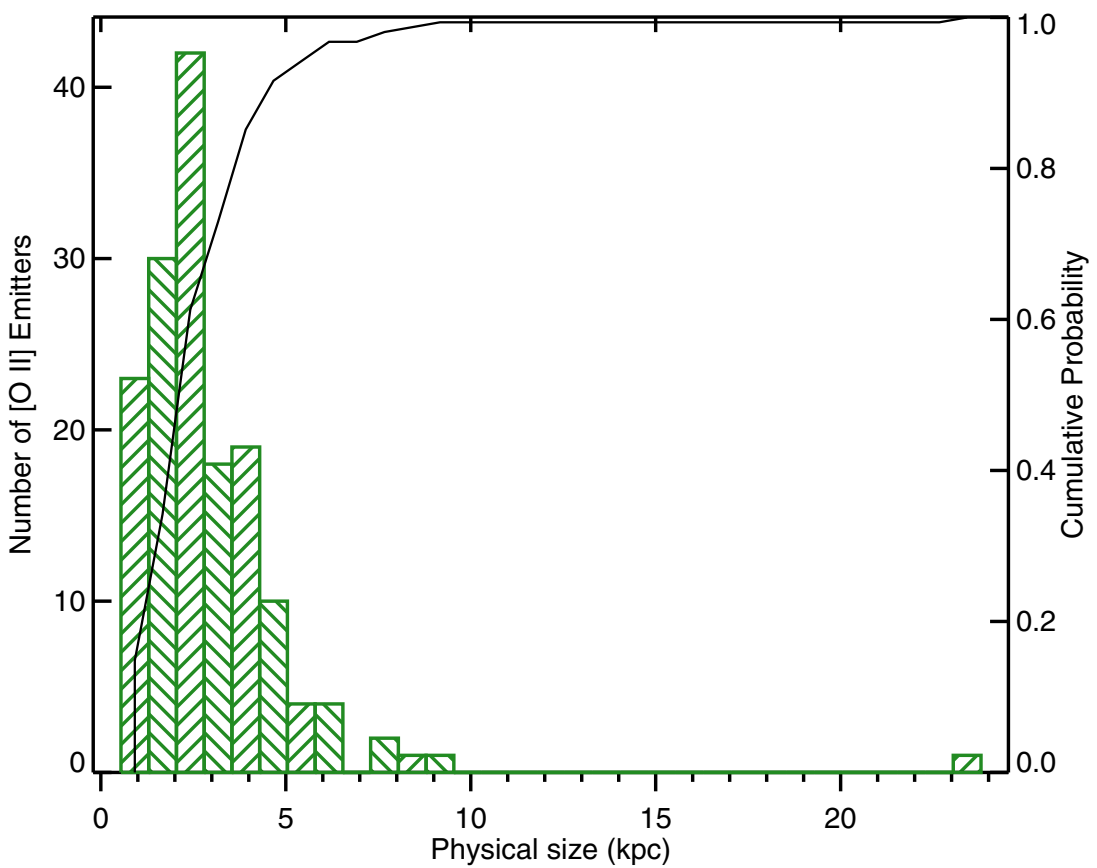

Figure 14. Physical half-light radii $\left(R_{50}\right)$ of the HPS [O II] emitters. The black line is the cumulative probability distribution. The median size is $R_{50}=2.43_{1.39}^{4.20} \mathrm{kpc}$ $\left(0.51_{0.27}^{0.87} \mathrm{arcsec}\right)$, where the upper and lower bounds are the 84 th and 16 th percentiles of the distribution, respectively.

selected galaxies in their sample fell above the line delineating quiescent from active galaxies. In particular, Figure 15 of Pirzkal et al. (2013) indicates that this effect is exaggerated for galaxies in which $\mathrm{H} \alpha$ and [O III] lines were observed. It should be noted that Pirzkal et al. measured their morphologies in the $\sim 4350 \AA$ rest frame, while our measurements (and those of Lotz et al. 2004) were performed using the F814W filter, i.e., the rest frame $R$ band. Morphological parameters can change depending on the image wavelength (Taylor-Mager et al. 2007), but since both bands are redward of the $4000 \AA$ break, this effect is not likely to be large. Still, it is possible that the difference between the [O II] galaxy samples are systematic. Additionally, despite the fact that the galaxies found by the PEARS survey and HPS are both emission-line selected, at any given redshift, the HPS galaxies extend to much higher masses (see Figure 7). This may point to differing star formation mechanisms at different masses, where higher mass galaxies are more likely to be undergoing normal star formation 
while lower mass galaxies are more likely to have disturbed morphologies.

While our [O II] emitters do not appear to be interacting, it is possible that correlations between stellar mass and morphology exist. Figure 13 shows $G$ and $M_{20}$ plotted as a function of both mass, SFR, and sSFR. Calculation of the Pearson's correlation coefficient (Pearson 1896) gives $r_{\mathrm{SFR}, M_{20}}=-0.17, r_{\mathrm{SFR}, G}=$ $0.016, r_{M, M_{20}}=-0.32, r_{M, G}=0.098, r_{\mathrm{sSFR}, M_{20}}=0.18$, and $r_{\mathrm{SSFR}, G}=-0.083$. These indicate no significant correlations between these parameters.

\section{SIZES}

In additional to quantifying their morphologies, we also measured the PSF-convolved physical sizes of our [O II] emitters by performing photometry using a series of circular apertures. Following Bond et al. (2009), we used these data to calculate each galaxy's half-light radius $\left(R_{50}\right)$ in a manner that is more robust than simple isophotal measurements. The distribution of physical sizes is shown in Figure 14. The [O II] emitters from this survey are fairly small, with a median size of $R_{50}=2.43_{1.39}^{4.20} \mathrm{kpc}$ $\left(0.51_{0.27}^{0.87}\right.$ arcseconds), where the upper and lower bounds are the 84th and 16th percentiles of the distribution, respectively. There are two obvious outliers: a galaxy at a redshift of $z=0.41$ with an angular size of $R_{50}=4 . .3$, and another at $z=0.09$ with $R_{50}=$ 3." 8 . These data have been excluded from our size analysis. Note that while the rest-frame bandpass of the $I$ filter changes with redshift, the range of redshifts considered here is fairly small and is unlikely to factor into the half-light radius results.

Figure 15 shows the sizes of these objects (in both kiloparsecs and arcseconds) as a function of redshift. There is no significant evolution in the physical sizes of these galaxies in this redshift range, as demonstrated by the scatter in the upper panel of Figure 15. The fact that very few large galaxies are seen at lower redshifts is simply a volume effect, as the probability of seeing these rare objects is roughly proportional to $(1+z)^{2}$. In the lower panel of Figure 15, the dashed lines show, from top to bottom, the 84 th, 50th, and 16 th percentiles in the given redshift bins. These lines indicate that there is little evidence of evolution in the galaxy sizes in this sample. The median physical size varies from $R_{50}=2.04 \pm 0.29 \mathrm{kpc}$ for redshifts $z<0.2$ to $R_{50}=2.61 \pm 0.56 \mathrm{kpc}$ in the redshift bin $0.5<z<0.6$. The two numbers are therefore well within the uncertainties of the measurements.

Pirzkal et al. (2006) examined low-mass emission-line galaxies in the Hubble Ultra Deep Field and found similar scatter in the evolution of sizes with redshift, noting that over the redshift range corresponding to that of HPS, the angular sizes of the eGRAPES increased by a factor of $\sim 2$, from $\sim 0^{\prime \prime} .2(1.33 \mathrm{kpc})$ at $z \sim 0.7$ to $\sim 00^{\prime \prime} .5(1.65 \mathrm{kpc})$ at $z \sim 2$ in the $4350 \AA$ rest frame. While we measure the HPS [O II] galaxy sizes using the $I$-band images (corresponding to $\sim R$-band rest frame), both data sets are redward of the $4000 \AA$ break, so there should not be a significant difference between the two bands. Additionally, van der Wel et al. (2014) examined the sizes of galaxies in the CANDELS/3D-HST fields using the HST/WFC3 IR bands of F125W, F140W, and F160W, just slightly redder than the $I$ band. For the median mass of the HPS sample $\left(M=9.20 M_{\odot}\right)$, they measure a median physical size of $R_{50}=3.09 \pm 0.07 \mathrm{kpc}$ for $z>0.5$ and $R_{50}=2.69 \pm 0.07 \mathrm{kpc}$ for $0.5<z<1$, suggesting slow to moderate size evolution between these epochs. These measurements, combined with our results, are consistent with previous studies of disk-dominated late-type galaxies that indi-

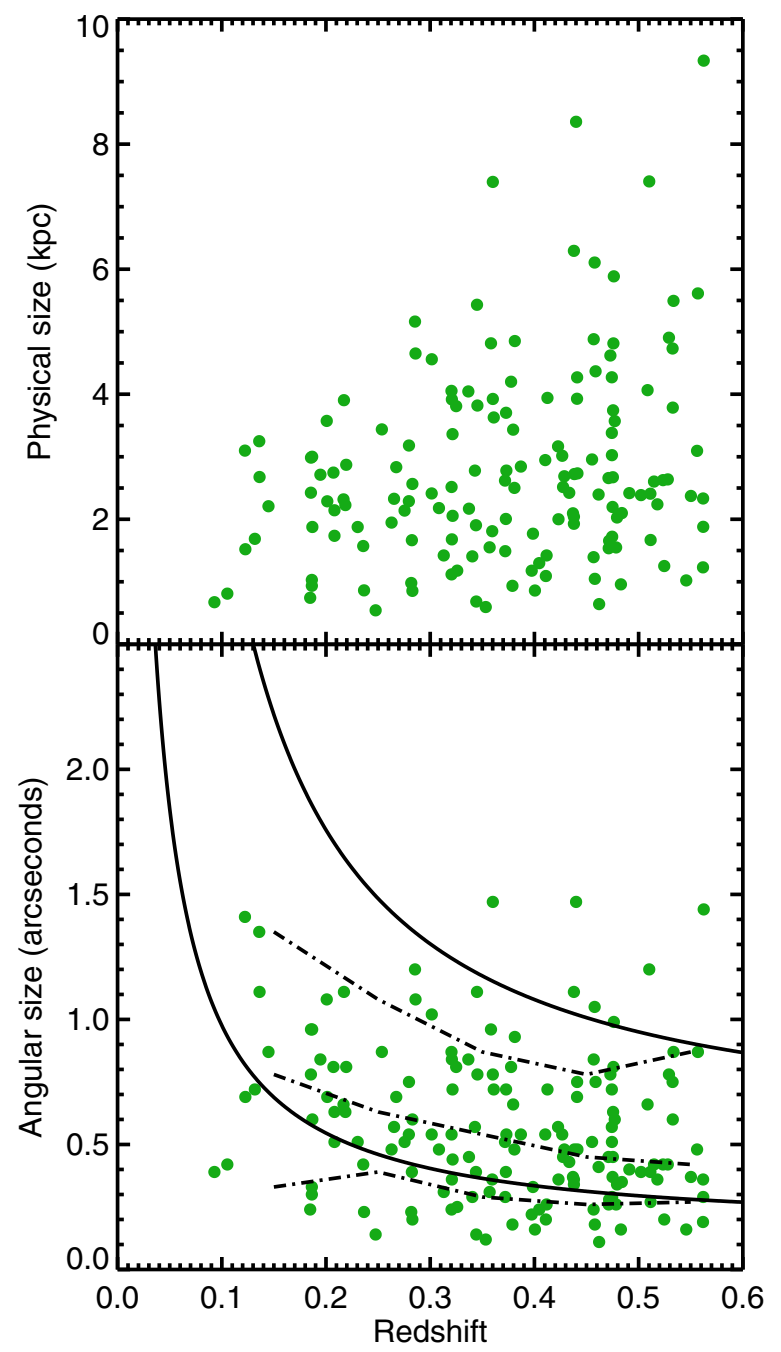

Figure 15. Physical sizes $\left(R_{50}\right)$ of the HPS [O II] emitters as a function of redshift. The solid black lines in the lower panel show what the angular size distribution would be assuming that the physical sizes of the galaxies do not evolve with redshift. The upper and lower lines represent the 84th and 16th percentiles of the size distribution at the highest sample redshift, corresponding to $R_{50}=0^{\prime \prime} .27$ and 0.87 (5.8 and $1.8 \mathrm{kpc}$ at $\left.z \sim 0.57\right)$, respectively. The black dash-dotted lines are, from top to bottom, the 84th, 50th, and 16th percentiles in the given redshift bins. The median error on the physical sizes, adapted from Lotz et al. (2006), are $\sim 0.1 \mathrm{kpc}$, falling almost within the size of the plot symbols.

cate that there is little, if any, size evolution at redshifts $z<1$ (Lilly et al. 1998; Ravindranath et al. 2004; Barden et al. 2005).

The size-mass distribution of the HPS [O II] emitters is show in Figure 16. As has been shown in previous studies (e.g., Shen et al. 2003; van der Wel et al. 2014), there is an obvious correlation between the mass of galaxies and their size. The slope of the relation, $\alpha=\log R_{50} / \log M_{*}=0.15 \pm 0.03$, is comparable to the $\alpha=0.22 \pm 0.03$ measurement for latetype galaxies in the CANDELS/3D-HST field (van der Wel et al. 2014), and midway between the values of $\alpha=0.14$ and 0.39 found for low- $\left(M_{*}<3.98 \times 10^{10} M_{\odot}\right)$ and highermass late-type galaxies in SDSS (Shen et al. 2003). Shen et al. (2003) showed that flatter slopes associated with the lower mass galaxies are consistent with models in which feedback driven by galactic winds overcomes the potential of the dark matter halos and suppress gas mass. Assuming that the specific angular momentum of a galaxy is similar to that of its halo, less-massive 


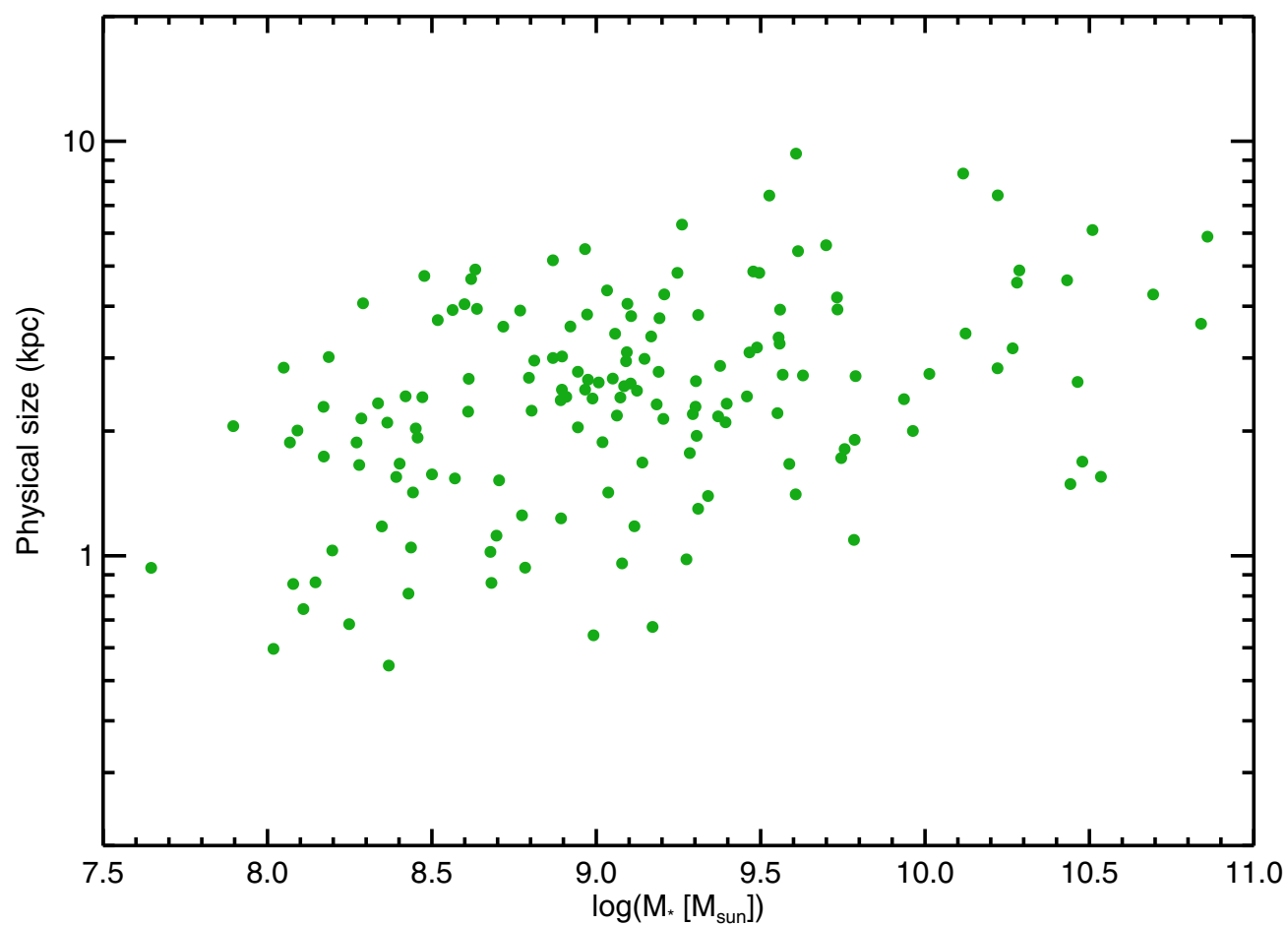

Figure 16. Physical sizes $\left(R_{50}\right)$ of the HPS [O II] emitters as a function of mass $\left(M_{*}\right)$. The slope of the size-mass relation is $\alpha=0.15$. The median error on the physical sizes, adapted from Lotz et al. (2006), are $\sim 0.1 \mathrm{kpc}$, falling almost within the size of the plot symbols.

late-type galaxies must have larger half-light radii, resulting in a flatter size-mass relation.

\section{CONCLUSIONS}

We presented an analysis of the physical properties of [O II] emitting galaxies within the HETDEX pilot survey with $z<0.57$. The data quality of this sample of galaxies allowed us to achieve a substantial improvement in the study of properties of star-forming galaxies in this redshift range. Stellar masses for these galaxies were determined by SED fitting. The observed SFR versus $M_{*}$ relation confirms the existence of the main sequence of galaxies, where SFRs decrease at lower masses and redshifts. The sSFRs increase for lower stellar mass galaxies, supporting the idea that the galaxies that are more efficiently forming stars in this redshift range are of lower masses, while the larger galaxies are ending their star formation. Examination of the morphologies of these galaxies gives no indication that the $[\mathrm{O} I \mathrm{II}]$ emitters are undergoing mergers, and there is no correlation between morphology parameters $\left(G\right.$ and $\left.M_{20}\right)$ and mass, SFR, or sSFR.

The HETDEX pilot survey has provided a test data set for the type of data produced in the main survey. HETDEX will provide upwards of $10^{6}[\mathrm{O}$ II] spectra, a data set that will serve to further refine the results presented here.

We wish to thank the anonymous reviewer for their invaluable comments. We acknowledge N. Pirzkal for generously sharing his data, and J. Lotz for making her morphology code available and advising in its use. Additionally, we are grateful to J. Adams and G. Blanc for providing the photometry from HPS. We thank the Cynthia and George Mitchell Foundation for funding the Mitchell Spectrograph, known formerly as VIRUS-P. J.S.B. is supported by the NSF Graduate Research Fellowship Program under grant DGE1255832. This work was also funded by the
NSF grant AST 09-26641. The Institute for Gravitation and the Cosmos is supported by the Eberly College of Science and the Office of the Senior Vice President for Research at The Pennsylvania State University. Computational support and resources were provided by the Research Computer and Cyberinfrastructure Unit of Information Technology Services at The Pennsylvania State University. This research has made use of NASA's Astrophysics Data System Bibliographic Services.

Facility: Smith (VIRUS-P)

\section{REFERENCES}

Abraham, R. G., Valdes, F., Yee, H. K. C., \& van den Bergh, S. 1994, ApJ, 432, 75

Acquaviva, V., Gawiser, E., \& Guaita, L. 2011, ApJ, 737, 47

Adams, J. J., Blanc, G. A., Hill, G. J., et al. 2011, ApJS, 192, 5

Barden, M., Rix, H.-W., Somerville, R. S., et al. 2005, ApJ, 635, 959

Bauer, A. E., Drory, N., Hill, G. J., \& Feulner, G. 2005, ApJL, 621, L89

Bauer, A. E., Hopkins, A. M., Gunawardhana, M., et al. 2013, MNRAS, 434, 209

Bershady, M. A., Jangren, A., \& Conselice, C. J. 2000, AJ, 119, 2645

Blanc, G. A., Adams, J. J., Gebhardt, K., et al. 2011, ApJ, 736, 31

Bond, N. A., Gawiser, E., Gronwall, C., et al. 2009, ApJ, 705, 639

Bouwens, R. J., Illingworth, G. D., Labbe, I., et al. 2011, Natur, 469, 504

Bruzual, G., \& Charlot, S. 2003, MNRAS, 344, 1000

Calzetti, D., Armus, L., Bohlin, R. C., et al. 2000, ApJ, 533, 682

Ciardullo, R., Gronwall, C., Adams, J. J., et al. 2013, ApJ, 769, 83

Ciardullo, R., Gronwall, C., Wolf, C., et al. 2012, ApJ, 744, 110

Conroy, C. 2013, ARA\&A, 51, 393

Conselice, C. J. 2003, ApJS, 147, 1

Cowie, L. L., Songaila, A., Hu, E. M., \& Cohen, J. G. 1996, AJ, 112, 839

Drory, N., Feulner, G., Bender, R., et al. 2001, MNRAS, 325, 550

Fumagalli, M., Patel, S. G., Franx, M., et al. 2012, ApJL, 757, L22

Garn, T., \& Best, P. N. 2010, MNRAS, 409, 421

Gelman, A., \& Rubin, D. B. 1992, StaSc, 7, 457

Giavalisco, M., Ferguson, H. C., Koekemoer, A. M., et al. 2004, ApJL, 600, L93

Gilbank, D. G., Baldry, I. K., Balogh, M. L., Glazebrook, K., \& Bower, R. G. 2010, MNRAS, 405, 2594

González, V., Labbé, I., Bouwens, R. J., et al. 2010, ApJ, 713, 115

Gronwall, C., Ciardullo, R., Hickey, T., et al. 2007, ApJ, 667, 79 
Hammer, F., Flores, H., Lilly, S. J., et al. 1997, ApJ, 481, 49

Heavens, A., Panter, B., Jimenez, R., \& Dunlop, J. 2004, Natur, 428, 625

Hill, G. J., Gebhardt, K., Komatsu, E., et al. 2008, in ASP Conf. Ser. 399 Panoramic Views of Galaxy Formation and Evolution, ed. T. Kodama, T. Yamada, \& K. Aoki (San Francisco, CA: ASP), 115

Hogg, D. W., Cohen, J. G., Blandford, R., \& Pahre, M. A. 1998, ApJ, 504, 622 Hopkins, A. M. 2004, ApJ, 615, 209

Jogee, S., Miller, S. H., Penner, K., et al. 2009, ApJ, 697, 1971

Kennicutt, R. C., \& Evans, N. J. 2012, ARA\&A, 50, 531

Kennicutt, R. C., Jr. 1998, ARA\&A, 36, 189

Kewley, L. J., Geller, M. J., \& Jansen, R. A. 2004, AJ, 127, 2002

Komatsu, E., Smith, K. M., Dunkley, J., et al. 2011, ApJS, 192, 18

Lara-López, M. A., Bongiovanni, A., Cepa, J., et al. 2010, A\&A, 519, A31

Lara-López, M. A., Hopkins, A. M., López-Sánchez, A. R., et al. 2013, MNRAS, 434, 451

Lewis, A., \& Bridle, S. 2002, PhRvD, 66, 103511

Lilly, S., Schade, D., Ellis, R., et al. 1998, ApJ, 500, 75

Lonsdale, C. J., Smith, H. E., Rowan-Robinson, M., et al. 2003, PASP, 115, 897

Lotz, J. M., Jonsson, P., Cox, T. J., et al. 2011, ApJ, 742, 103

Lotz, J. M., Madau, P., Giavalisco, M., Primack, J., \& Ferguson, H. C. 2006, ApJ, 636, 592

Lotz, J. M., Primack, J., \& Madau, P. 2004, AJ, 128, 163

Martin, D. C., Fanson, J., Schiminovich, D., et al. 2005, ApJL, 619, L1

Meurer, G. R., Heckman, T. M., \& Calzetti, D. 1999, ApJ, 521, 64
Noeske, K. G., Weiner, B. J., Faber, S. M., et al. 2007, ApJL, 660, L43

Pearson, K. 1896, RSPTA, 187, 253

Pettini, M., Shapley, A. E., Steidel, C. C., et al. 2001, ApJ, 554, 981

Pierre, M., Valtchanov, I., Altieri, B., et al. 2004, JCAP, 09, 011

Pirzkal, N., Rothberg, B., Ly, C., et al. 2013, ApJ, 772, 48

Pirzkal, N., Rothberg, B., Nilsson, K. K., et al. 2012, ApJ, 748, 122

Pirzkal, N., Xu, C., Ferreras, I., et al. 2006, ApJ, 636, 582

Ravindranath, S., Ferguson, H. C., Conselice, C., et al. 2004, ApJL, 604, L9

Robaina, A. R., Bell, E. F., van der Wel, A., et al. 2010, ApJ, 719, 844

Salpeter, E. E. 1955, ApJ, 121, 161

Sanders, D. B., Salvato, M., Aussel, H., et al. 2007, ApJS, 172, 86

Scoville, N., Aussel, H., Brusa, M., et al. 2007, ApJS, 172, 1

Searle, L., Sargent, W. L. W., \& Bagnuolo, W. G. 1973, ApJ, 179, 427

Serra, P., Amblard, A., Temi, P., et al. 2011, ApJ, 740, 22

Shen, S., Mo, H. J., White, S. D. M., et al. 2003, MNRAS, 343, 978

Taylor-Mager, V. A., Conselice, C. J., Windhorst, R. A., \& Jansen, R. A. 2007, ApJ, 659, 162

van der Wel, A., Franx, M., van Dokkum, P. G., et al. 2014, ApJ, 788, 28

Whitaker, K. E., van Dokkum, P. G., Brammer, G., \& Franx, M. 2012, ApJL, 754, L29

Wolf, C., Bell, E. F., McIntosh, D. H., et al. 2005, ApJ, 630, 771

Wuyts, S., Franx, M., Cox, T. J., et al. 2009, ApJ, 696, 348

Zahid, H. J., Kashino, D., Silverman, J. D., et al. 2014, ApJ, 792, 75 